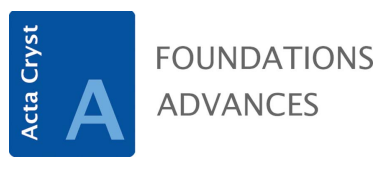

ISSN 2053-2733

\title{
Group-theoretical analysis of 1:3 A-site-ordered perovskite formation
}

\author{
Mikhail V. Talanov* \\ Southern Federal University, Rostov-on-Don, Russian Federation. *Correspondence e-mail: mvtalanov@gmail.com
}

Received 25 June 2018

Accepted 26 December 2018

Edited by W. F. Kuhs, Georg-August University Göttingen, Germany

Keywords: quadruple 1:3 A-site-ordered perovskites; group-theoretical analysis; low-symmetry phases; full set of order parameters; tilts of octahedra; archetype structure.

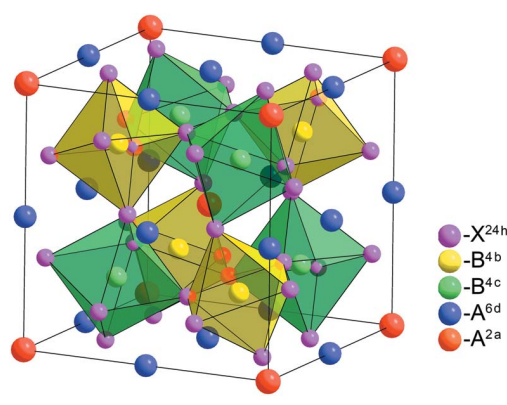

OPEN $\odot$ ACCESS
The quadruple perovskites $A A^{\prime}{ }_{3} B_{4} X_{12}$ are characterized by an extremely wide variety of intriguing physical properties, which makes them attractive candidates for various applications. Using group-theoretical analysis, possible 1:3 $A$-siteordered low-symmetry phases have been found. They can be formed from a parent $P m \overline{3} m$ perovskite structure (archetype) as a result of real or hypothetical (virtual) phase transitions due to different structural mechanisms (orderings and displacements of atoms, tilts of octahedra). For each type of low-symmetry phase, the full set of order parameters (proper and improper order parameters), the calculated structure, including the space group, the primitive cell multiplication, splitting of the Wyckoff positions and the structural formula were determined. All ordered phases were classified according to the irreducible representations of the space group of the parent phase (archetype) and systematized according to the types of structural mechanisms responsible for their formation. Special attention is paid to the structural mechanisms of formation of the low-symmetry phase of the compounds known from experimental data, such as: $\mathrm{CaCu}_{3} \mathrm{Ti}_{4} \mathrm{O}_{12}, \mathrm{CaCu}_{3} \mathrm{Ga}_{2} \mathrm{Sn}_{2} \mathrm{O}_{12}, \mathrm{CaMn}_{3} \mathrm{Mn}_{4} \mathrm{O}_{12}$, $\mathrm{Ce}_{1 / 2} \mathrm{Cu}_{3} \mathrm{Ti}_{4} \mathrm{O}_{12}, \mathrm{LaMn}_{3} \mathrm{Mn}_{4} \mathrm{O}_{12}, \mathrm{BiMn}_{3} \mathrm{Mn}_{4} \mathrm{O}_{12}$ and others. For the first time, the phenomenon of variability in the choice of the proper order parameters, which allows one to obtain the same structure by different group-theoretical paths, is established. This phenomenon emphasizes the fundamental importance of considering the full set of order parameters in describing phase transitions. Possible transition paths from the archetype with space group $\operatorname{Pm} \overline{3} m$ to all 1:3 $A$-site-ordered perovskites are illustrated using the Bärnighausen tree formalism. These results may be used to identify new phases and interpret experimental results, determine the structural mechanisms responsible for the formation of low-symmetry phases as well as to understand the structural genesis of the perovskite-like phases. The obtained non-model group-theoretical results in combination with crystal chemical data and first-principles calculations may be a starting point for the design of new functional materials with a perovskite structure.

\section{Introduction}

$A$-site-ordered quadruple $A A^{\prime}{ }_{3} B_{4} X_{12}$ perovskites occupy a special place among a large variety of functional materials (Mitchel, 2002; Tilley, 2016; King \& Woodward, 2010; Aleksandrov \& Beznosikov, 1997; Shimakawa, 2008; Yamada, 2017; Long, 2016; Vasil'ev \& Volkova, 2007). This numerous family of materials is characterized by intriguing physical properties such as a giant dielectric constant (Subramanian et al., 2000; Homes et al., 2001), positive and negative magnetodielectricity (Imamura et al., 2008), low- and high-field magnetoresistance (Chen et al., 2014; Kida et al., 2012), multiferroic properties (Wang et al., 2015; Zhou et al., 2017), large negative and positive thermal expansion (Long, Hayashi et al., 2009; Zhang et al., 2014; Long \& Shimakawa, 2010) and heavy Fermion-like behavior (Kobayashi et al., 2004). 
1:3 $A$-site-ordered perovskites were discovered in 1967 (Deschanvres et al., 1967). Interest in this class of materials increased sharply 33 years after the discovery in $\mathrm{CaCu}_{3} \mathrm{Ti}_{4} \mathrm{O}_{12}$ of a giant low-frequency dielectric constant $\left(\varepsilon_{0} \simeq 10^{5}\right)$, which is stable over a wide temperature range from 100 to $600 \mathrm{~K}$ (Subramanian et al., 2000; Homes et al., 2001). This discovery allowed $\mathrm{CaCu}_{3} \mathrm{Ti}_{4} \mathrm{O}_{12}$ to be considered as a possible material for devices, in particular in elements of static and dynamic random access memory. The giant dielectric constant in $\mathrm{CaCu}_{3} \mathrm{Ti}_{4} \mathrm{O}_{12}$ is usually associated with an extrinsic contribution, in particular with the internal barrier layer capacitance (Sinclair et al., 2002; Cohen et al., 2003; Tselev et al., 2004; Adams et al., 2006). This is due to the presence of a heterogeneous microstructure in the sample (semiconducting grains separated by insulating grain boundaries), which results in Maxwell-Wagner relaxation. Despite the extrinsic mechanism of the appearance of the giant dielectric constant in $\mathrm{CaCu}_{3} \mathrm{Ti}_{4} \mathrm{O}_{12}$, widely discussed in the literature, a number of works emphasize possible intrinsic contributions to the dielectric constant value (Liu et al., 2005; Zhu et al., 2007).

The discovery of the temperature-induced $A-B$ intersite charge transfer effect, which is associated with large negative thermal expansion in $\mathrm{LaCu}_{3} \mathrm{Fe}_{4} \mathrm{O}_{12}$, aroused great interest (Long, Hayashi et al., 2009). Later, charge transfer between the different transition-metal cations at the square-planar $A^{\prime}$ and octahedral $B$ sites was found in other 1:3 $A$-site-ordered perovskites: $\mathrm{BiCu}_{3} \mathrm{Fe}_{4} \mathrm{O}_{12}$ (Long \& Shimakawa, 2010; Long, Saito et al., 2009), $\mathrm{LaCu}_{3} \mathrm{Cr}_{4} \mathrm{O}_{12}$ and $\mathrm{YCu}_{3} \mathrm{Cr}_{4} \mathrm{O}_{12}$ (Zhang et al., 2014). The latter two compounds showed positive thermal expansion-like volume changes at the intersite charge transfer transition. These materials can be used in such applications as elasticity-tuned sensors and switching devices (Long, Hayashi et al., 2009). Note the site-selective electron doping effect, which is associated with a change in the valence in either the $A^{\prime}$ or the $B$ sites, depending on the choice of the type of atom in the $A$ site (Zhang, Saito, Mizumaki et al., 2013).

A wide variety of magnetic properties is also characteristic of 1:3 $A$-site-ordered perovskites which causes considerable scientific interest in these materials. For example, $\mathrm{CaCu}_{3^{-}}$ (Ge,Sn) ${ }_{4} \mathrm{O}_{12}$ and (La,Dy) $\mathrm{Cu}_{3}\left(\mathrm{Ge}_{3 / 4} \mathrm{Ga}_{1 / 4}\right)_{4} \mathrm{O}_{12}$ are ferromagnets (Shimakawa, 2008; Shiraki et al., 2007), $\mathrm{CaMn}_{3} \mathrm{~V}_{4} \mathrm{O}_{12}$ and $\mathrm{YMn}_{3} \mathrm{Al}_{4} \mathrm{O}_{12}$ are antiferromagnets (Zhang, Saito, Chen et al., 2013; Toyoda et al., 2015), $\mathrm{CaCu}_{3} \mathrm{Fe}_{4} \mathrm{O}_{12}, \mathrm{Ce}_{1 / 2} \mathrm{Cu}_{3} \mathrm{Ti}_{4} \mathrm{O}_{12}$, $\mathrm{BiCu}_{3} \mathrm{Mn}_{4} \mathrm{O}_{12}$ and $\mathrm{CaCu}_{3} \mathrm{Fe}_{2} \mathrm{Re}_{2} \mathrm{O}_{12}$ are ferrimagnets (Chen et al., 2014; Yamada et al., 2008; Saito et al., 2014; Takata et al., 2007), ( $\mathrm{Na}, \mathrm{Ca}, \mathrm{Y}) \mathrm{Cu}_{3} \mathrm{~V}_{4} \mathrm{O}_{12}$ are Pauli paramagnetics (Shimakawa, 2008; Zhang, Saito, Mizumaki et al., 2013], and $\mathrm{Ca}\left(\mathrm{Mn}_{x} \mathrm{Cu}_{1-x}\right)_{3} \mathrm{~V}_{4} \mathrm{O}_{12}$ and $\mathrm{LuMn}_{3} \mathrm{~V}_{4} \mathrm{O}_{12}$ are spin-glass phases (Zhang, Saito, Chen et al., 2013; Shimakawa et al., 2014). The magnetic properties of these materials can be tuned both by changing the chemical composition (Shimakawa, 2008) and by cation (or vacancy) ordering (Saito et al., 2014; Shimakawa et al., 2014). An unusual geometric frustrated state in the $\mathrm{Fe}^{3+}$ spin sublattice was found in the $A$ - and $B$-site-ordered quadruple perovskite $\mathrm{CaCu}_{3} \mathrm{Fe}_{2} \mathrm{Sb}_{2} \mathrm{O}_{12}$ (Chen et al., 2014). The magnetic and transport properties of $\mathrm{CaCu}_{3} \mathrm{Ru}_{4} \mathrm{O}_{12}$ have attracted attention due to their heavy Fermion-like behavior
(Kobayashi et al., 2004). $\mathrm{NaMn}_{3} \mathrm{Mn}_{4} \mathrm{O}_{12}$ shows a sequence of phase transitions associated with the spin, charge and orbital orderings (Prodi et al., 2004). Recently, a strong spindriven magnetoelectric effect was observed in a perovskite $\mathrm{LaMn}_{3} \mathrm{Cr}_{4} \mathrm{O}_{12}$ with a centrosymmetric cubic structure (Wang et al., 2015). It is assumed that ferroelectricity in this compound is of an electronic nature and is formed due to magnetic ordering in two magnetic sublattices.

Thanks to all these physical properties, 1:3 $A$-site-ordered quadruple $A A^{\prime}{ }_{3} B_{4} X_{12}$ perovskites are still of great interest. The diverse and unique physical properties of 1:3 $A$-siteordered perovskites are mainly due to the ordered structure of these crystals. We have previously carried out a study of cation- and anion-ordered low-symmetry modifications of the parent $P m \overline{3} m$ perovskite structure (Talanov, Shirokov et al., 2014; Talanov, Talanov et al., 2014; Talanov et al., 2016). In this article, we emphasize the systematic study of perovskite-like structures with an order of 1:3 in the $A$ sublattice which are formed as a result of real or hypothetical (virtual) structural phase transitions from the parent phase through various structural mechanisms.

Currently, there are two main theoretical approaches to the calculation of possible structures of the phases. The first approach is based on the International Tables for Crystallography (2010). We will call this the ITC approach for short. The ITC approach is described in detail by Bärnighausen (1975, 1980), Müller (2004, 2013). The second approach is based on group-theoretical methods of the Landau theory of phase transitions (Landau, 1937). It is based on the theory of irreducible representations (irreps) of the space symmetry groups. We will call it the 'representation' approach, or the R-approach. Here we describe briefly each of these approaches.

The ITC approach accumulates crystallographic knowledge gained over more than 100 years - since the discovery of space symmetry groups by Fedorov (1891) and Schoenflies (1891). The visual representation of symmetry relations between different structures/phases is given in the form of a hierarchical 'family tree' or Bärnighausen tree. The special Bärnighausen formalism is proposed, including information about the paths of transition from the initial highsymmetry structures/phases (aristotype) with space group $G$ to 'daughter' low-symmetry structures/phases (hettotypes) with space group $H$ indicating the kind of maximum subgroup and index of the symmetry reduction. According to the Hermann theorem (Hermann, 1928) there are two kinds of maximal subgroups: isotranslational (now called 'translationengleiche', i.e. subgroups $H$ have the same translation lattice as $G$ ) and isoclass (their name is 'klassengleiche', i.e. subgroups $H$ having a different translation lattice, but belonging to the same crystal class as $G$ ). In the Bärnighausen formalism these subgroups are denoted by ' $t n$ ' and ' $k n$ ', respectively, where $n$ is an index of symmetry reduction (Müller, 2013). From the kinds of subgroups it is possible to deduce what and how many kinds of domains can result from a phase transition or topotactic reaction (Lotgering, 1959; Giovanoli \& Leuenberger, 1969) involving a symmetry reduction. In addition, 
changes in the basis vectors, the origin shift and the splitting of Wyckoff positions are indicated. Numerous examples of the application of the ITC approach are given by Müller (2013) and Bärnighausen (1980). This approach is a useful tool for solving the structure of new crystals as well as for the structural design of novel materials.

The R-approach has been widely used in the investigation of low-symmetry structures of crystals and structural mechanisms of phase transitions ever since the classical works of Landau and Lifshitz (Landau, 1937; Lifshitz, 1941; Landau \& Lifshitz, 1976). It consists of two stages. The first stage includes finding all possible low-symmetry phases, corresponding order parameters $(\mathrm{OPs})$, basic vectors of primitive cells and changing of primitive cell volume $\left(V / V_{0}\right)$ for the irrep of the space group in the parent phase. At the second stage, the structures of the low-symmetry phases are determined. This problem was examined by Sakhnenko et al. (1986) and a general method for its solution was suggested there. The structure of the low-symmetry phase is connected with the socalled 'complete condensate of OPs' [the full set of the proper (primary) and the improper (secondary) OPs] as well as with the basis functions of irreps.

The group-theoretical analysis is quite a cumbersome procedure requiring the use of complex computer programs especially if OPs are multicomponent. There are some software applications for solution of the group-theoretical tasks: CONDENSATE and BASIS (Chechin, 1989), ISOTROPY (Howard \& Stokes, 2005; Stokes \& Hatch, 2002), the Bilbao Crystallographic Server (Aroyo et al., 2006; Perez-Mato et al., 2010) and others.

Visualization of the crystallographic analysis results of new structures is an attractive part of the ITC approach. This is used in the routine practice of solving structures. The $\mathrm{R}$-approach is less visual, but it allows one to describe and predict not only atom but also spin and orbital structures, as well as predict important physical effects, revealing the physical nature of proper and improper OPs. This approach is naturally linked with phenomenological thermodynamics, quantum mechanics, optics, magnetism, orbital physics and other fields of science.

The use of only one of the above approaches (ITC or R) considerably reduces the possibilities of applying the obtained results to solving various theoretical and practical problems of crystallography and other sciences associated with it. These two approaches complement each other. In our study of 1:3 $A$-site-ordered perovskite-like structures, an emphasis will be placed on the R-approach. But in the final part of the article, the Bärnighausen formalism will be used to visualize the genesis of 1:3 $A$-ordered perovskite-like structures and to establish the relationship between the ITC and R-approaches.

C. J. Howard and H. T. Stokes were the first to show that the 1:3 $A$-site ordering is associated with an $\mathrm{OP}$, transforming by the irrep $\mathbf{k}_{11} \tau_{1}\left(M_{1}^{+}\right)$(Howard \& Stokes, 2005). This study relies on the above work and supplements it. It will be shown that 1:3 $A$-site-ordered low-symmetry phases can be formed in different ways. A group-theoretical analysis of the structural mechanisms of formation of 1:3 $A$-site-ordered low-symmetry phases, as well as a group-theoretical map of their generation paths, are reported.

In this work, the possible structures of $A$-site-ordered quadruple $A A^{\prime}{ }_{3} B_{4} X_{12}$ perovskites have been found based on powerful group-theoretical methods of Landau phase transition theory (Howard \& Stokes, 2005; Howard et al., 2003; Aroyo et al., 2006; Perez-Mato et al., 2010; Toledano \& Toledano, 1987; Toledano \& Dmitriev, 1996; Birman, 1978; Vinberg et al., 1974; Aleksandrov \& Bartolomé, 2001; Bock \& Müller, 2002; Balachandran \& Rondinelli, 2013; Stokes \& Hatch, 1988; Chechin, 1989; Talanov et al., 2015, 2018; Talanov \& Shirokov, 2014; Talanov, 2007, 2018; Stokes \& Campbell, 2017). The ISOTROPY software suite was used for the calculations (Howard \& Stokes, 1998, 2004, 2005; Campbell et al., 2006). In particular, the ISOSUBGROUP program was used to obtain the list of low-symmetry phases induced by various OPs, as well as to determine the improper OPs. For a detailed study of the low-symmetry phase structure (splitting of the Wyckoff positions, obtaining different domains of the same phase etc.), the ISOTROPY program and ITC (2010) were used.

Thus, the aim of this study is a group-theoretical analysis of possible pathway formation and structural genesis of 1:3 $A$-site-ordered perovskites using the ITC and R-approaches.

\section{Paths of 1:3 A-site-ordered perovskite formation}

The space group of the $A B X_{3}$ cubic perovskite structure is $P m \overline{3} m$. In this structure, $A$ cations occupy Wyckoff position $1 a$ with cubo-octahedral coordination and with local symmetry $m \overline{3} m$, octahedral $B$ cations occupy Wyckoff position $1 b$ with local symmetry $m \overline{3} m$ and $X$ anions occupy Wyckoff position $3 c$ with local symmetry $4 / \mathrm{mm} . \mathrm{m}$. Perovskites are referred to as anion-octahedral-type structures, i.e. $B X_{6}$ octahedra act as building blocks which are connected together by common vertices in three directions and are separated by $A$ cations located in cubo-octahedral voids. Note that $1 a$ and $1 b$ positions in the perovskite structure are symmetrically equivalent. They are connected by an external automorphism, i.e. origin shift $\left(\frac{1}{2} \frac{1}{2} \frac{1}{2}\right)$ of the unit cell. This means that the origin can be chosen both at $A$ - (like in this work) and $B$-cation sites. The fact that the irreps responsible for these two descriptions are different must be taken into account.

The idealized perovskite structure is the archetype (this word originates from the ancient Greek one ' $\alpha \rho \rho \chi \varepsilon \tau v \pi o v$ ' which means 'prototype') and/or aristotype (from the ancient Greek word ' $\alpha \rho \iota \sigma \tau o \xi$ ', 'highest') for low-symmetry modifications (Megaw, 1957, 1973; Aleksandrov et al., 1981; Talanov et al., 2016). ${ }^{1}$ Atom displacements (including ordered tilts or rotations of the $B X_{6}$ octahedra) and orderings, as well as their combinations, result in lower-symmetry superstructures.

\footnotetext{
${ }^{1}$ In this article, we consider the ideal perovskite structure as an archetype, and ideal cation and anion superstructures of perovskites as aristotypes [see details on this issue in the work of Talanov et al. (2016)]. By definition, groupsubgroup relations necessarily exist between the space groups of the parent structure (archetype and aristotype) and the observed one. This can then be qualified as distorted (and/or ordered) structures and can be described as the parent crystalline structure plus a static symmetry-breaking structural distortion (and/or ordering).
} 
Table 1

Physical realization of the OP connected with atom non-polar and polar displacements, atom orderings and tilts of octahedra in perovskite structure (the unit irrep is not shown).

\begin{tabular}{|c|c|c|c|c|}
\hline \multirow[b]{2}{*}{ Physical realization } & \multicolumn{4}{|l|}{ Wavevectors } \\
\hline & $\mathbf{k}_{10}\left(\begin{array}{lll}0 & 0 & \frac{1}{2}\end{array}\right)(X)$ & $\mathbf{k}_{11}\left(0 \frac{1}{2} \frac{1}{2}\right)(M)$ & $\mathbf{k}_{12}\left(\begin{array}{lll}0 & 0 & 0\end{array}\right)(\Gamma)$ & $\mathbf{k}_{13}\left(\frac{1}{2} \frac{1}{2} \frac{1}{2}\right)(R)$ \\
\hline \multicolumn{5}{|c|}{ Non-polar displacement } \\
\hline$X$-site $\left(D_{X}\right)$ & $\begin{array}{l}\tau_{1}\left(X_{1}^{+}\right)+\tau_{4}\left(X_{3}^{-}\right)+\tau_{6}\left(X_{2}^{-}\right) \\
\quad+2 \tau_{9}\left(X_{5}^{+}\right)+\tau_{10}\left(X_{5}^{-}\right)\end{array}$ & $\begin{array}{l}\tau_{1}\left(M_{1}^{+}\right)+\tau_{3}\left(M_{3}^{+}\right)+\tau_{5}\left(M_{2}^{+}\right)+\tau_{6}\left(M_{2}^{-}\right) \\
\quad+\tau_{7}\left(M_{4}^{+}\right)+\tau_{9}\left(M_{5}^{+}\right)+\tau_{10}\left(M_{5}^{-}\right)\end{array}$ & $\tau_{8}\left(\Gamma_{5}^{-}\right)$ & $\begin{array}{l}\tau_{1}\left(R_{2}^{-}\right)+\tau_{6}\left(R_{3}^{-}\right) \\
\quad+\tau_{8}\left(R_{5}^{-}\right)+\tau_{10}\left(R_{4}^{-}\right)\end{array}$ \\
\hline \multicolumn{5}{|l|}{ Ordering } \\
\hline$A$-site $\left(O_{A}\right)$ & $\tau_{1}\left(X_{1}^{+}\right)$ & $\tau_{1}\left(M_{1}^{+}\right)$ & $\tau_{1}\left(\Gamma_{1}^{+}\right)$ & $\tau_{1}\left(R_{1}^{+}\right)$ \\
\hline$B$-site $\left(O_{B}\right)$ & $\tau_{4}\left(X_{3}^{-}\right)$ & $\tau_{7}\left(M_{4}^{+}\right)$ & $\tau_{1}\left(\Gamma_{1}^{+}\right)$ & $\tau_{4}\left(R 2^{-}\right)$ \\
\hline$X$-site $\left(O_{X}\right)$ & $\tau_{1}\left(X_{1}^{+}\right)+\tau_{4}\left(X_{3}^{-}\right)+\tau_{8}\left(X_{4}^{-}\right)$ & $\tau_{7}\left(M_{4}^{+}\right)+\tau_{10}\left(M_{5}^{-}\right)$ & $\tau_{1}\left(\Gamma_{1}^{+}\right)+\tau_{5}\left(\Gamma_{3}^{+}\right)$ & $\tau_{7}\left(R_{5}^{+}\right)$ \\
\hline \multicolumn{5}{|c|}{ Tilts or tilt-like distortions } \\
\hline
\end{tabular}

In the Landau theory, the low-symmetry structure formation is described by proper and improper OPs, which are transformed according to the irrep of the space symmetry group of the high-symmetry parent phase. Irreps (according to which a proper OP is transformed) determine the symmetry and structural motif of low-symmetry phases near the transition point. However, when a low-symmetry structure is far from the temperature of the phase transition $\left(T_{\mathrm{c}}\right)$, the contribution of improper OPs to atom displacements (and/or orderings) can become essential (Dimmock, 1963; Bruce \& Cowley, 1981; Sakhnenko et al., 1986; Molokeev \& Misyul', 2012). The appearance of these contributions is connected with nonlinear interactions between different degrees of freedom in a crystal. There are two main notation schemes of irreps: by Kovalev and by Miller-Love. ${ }^{2}$ In the first case, the designation is $\mathbf{k}_{n} \tau_{m}$, where $n$ is the number of the wavevector $\mathbf{k}$ and $m$ is the number of the corresponding irrep. In the second case, the wavevectors are denoted by different capital letters $(\Gamma, M, X \ldots)$, and numbers of irreps are designated as subscript numbers and superscript signs ' + ' and '-'. We will use the designations for irreps according to both said schemes. In addition, the OP direction in the OP space is necessary to indicate its unambiguous identification. The OPs transformed by irreps with different $\mathbf{k}$ values will be denoted by various Greek letters $(\eta, \varphi, \sigma, \Psi)$. In this article, we will consider only those irreps that satisfy the Lifshitz criterion (Landau \& Lifshitz, 1976), i.e. they induce commensurate phases.

The full set of proper and improper OPs fully determines the structure and all possible symmetry-dependent properties of the crystal (Sakhnenko et al., 1986). The analysis of the full

\footnotetext{
2 The correspondence of designations by Kovalev (1993) and Miller \& Love (1967) is as follows. For $\mathbf{k}_{10}(X): \tau_{1}\left(X_{1}^{+}\right), \tau_{2}\left(X_{1}{ }^{-}\right), \tau_{3}\left(X_{3}^{+}\right), \tau_{4}\left(X_{3}{ }^{-}\right), \tau_{5}\left(X_{2}{ }^{+}\right)$, $\tau_{6}\left(X_{2}{ }^{-}\right), \tau_{7}\left(X_{4}^{+}\right), \tau_{8}\left(X_{4}^{-}\right), \tau_{9}\left(X_{5}^{+}\right), \tau_{10}\left(X_{5}{ }^{-}\right)$. For $\mathbf{k}_{11}(M): \tau_{1}\left(M_{1}{ }^{+}\right), \tau_{2}\left(M_{1}{ }^{-}\right)$, $\tau_{3}\left(M_{3}{ }^{+}\right), \tau_{4}\left(\mathrm{M}_{3}{ }^{-}\right), \tau_{5}\left(M_{2}{ }^{+}\right), \tau_{6}\left(M_{2}{ }^{-}\right), \tau_{7}\left(M_{4}{ }^{+}\right), \tau_{8}\left(M_{4}{ }^{-}\right), \tau_{9}\left(M_{5}{ }^{+}\right), \tau_{10}\left(M_{5}{ }^{-}\right)$. For $\mathbf{k}_{12}(\Gamma): \tau_{1}\left(\Gamma_{1}^{+}\right), \tau_{2}\left(\Gamma_{1}^{-}\right), \tau_{3}\left(\Gamma_{2}^{+}\right), \tau_{4}\left(\Gamma_{2}^{-}\right), \tau_{5}\left(\Gamma_{3}^{+}\right), \tau_{6}\left(\Gamma_{3}^{-}\right), \tau_{7}\left(\Gamma_{5}^{+}\right), \tau_{8}\left(\Gamma_{5}{ }^{-}\right)$, $\tau_{9}\left(\Gamma_{4}^{+}\right), \tau_{10}\left(\Gamma_{4}^{-}\right)$. For $\mathbf{k}_{13}(R): \tau_{1}\left(R_{1}^{+}\right), \tau_{2}\left(R_{1}^{-}\right), \tau_{3}\left(R_{2}^{+}\right), \tau_{4}\left(R_{2}^{-}\right), \tau_{5}\left(R_{3}^{+}\right)$, $\tau_{6}\left(R_{3}{ }^{-}\right), \tau_{7}\left(R_{5}^{+}\right), \tau_{8}\left(R_{5}{ }^{-}\right), \tau_{9}\left(R_{4}^{+}\right), \tau_{10}\left(R_{4}^{-}\right)$.
}

set of the OPs allows conclusions to be drawn about the nature of the crystal properties. The proper OPs that are responsible for the manifestation of ferroelectric properties, orderings and atom displacements, tilts of anion octahedra and Jahn-Teller distortions in perovskites are well known (Howard \& Stokes, 1998, 2004, 2005; Howard et al., 2003; Carpenter \& Howard, 2009; Howard \& Carpenter, 2010; Senn \& Bristowe, 2018; Talanov, Shirokov et al., 2014; Talanov, Talanov et al., 2014).

In Table 1, irreps according to which OPs are transformed inducing atom displacements ${ }^{3}$ (polar and non-polar), atom orderings ${ }^{4}$ and tilts of anion octahedra (or tilt-like distortions) in perovskites are summarized.

Let us return to the analysis of the ways of 1:3 $A$-ordered phase formation (Fig. 1). Irrep $\mathbf{k}_{11} \tau_{1}\left(M_{1}^{+}\right)$may in different ways enter into a full set of OPs, describing different mechanisms of 1:3 $A$-site-ordered phase formation. These OPs can be either proper or improper OPs. In addition, they can form combinations with other OPs. Thus, there are two types of mechanism of 1:3 $A$-site-ordered perovskite formation associated with proper and improper atom orderings. For both mechanisms, further analysis is connected with the number of proper OPs. For mechanisms of the first type, proper ordering in perovskite $A$ sublattices and its combinations with other OPs are considered. For mechanisms of the second type, tilts of anion octahedra, atom ordering in the $X$ sublattice, as well as combinations of these mechanisms with ordering in the $B$ sublattice and atom displacements, are considered.

To enumerate all the low-symmetry phases formed as a result of atom displacements, it is necessary to use the OPs

\footnotetext{
${ }^{3}$ A set of space-group irreps of the parent cubic perovskite phase, basic functions of which make it possible to determine the atom displacements (including non-polar and polar displacements) at $1 a, 1 b$ and $3 c$ Wyckoff positions of the $\operatorname{Pm} \overline{3} m$ space group, is a mechanical (or atom-displacement) representation of the perovskite structure.

${ }^{4} \mathrm{~A}$ set of space-group irreps of the parent cubic perovskite phase, basic functions of which make it possible to determine the atom permutations at $1 a$, $1 b$ and $3 c$ Wyckoff positions of the $\operatorname{P} m \overline{3} m$ space group, is a permutational representation of the perovskite structure.
} 
that are transformed by the irreps taken from the composition of the mechanical (atom-displacement) representation of the perovskite structure. This representation includes 20 different irreps (Table 1), combinations of which induce more than 1200 low-symmetry phases. Because of the cumbersome nature of the complete list of all low-symmetry phases formed as a result of atom displacements, the study is limited to irrep $\mathbf{k}_{12} \tau_{10}\left(\Gamma_{4}^{-}\right)$only. This irrep is associated with the polar displacements of atoms. The research focuses on phases with polar displacements, because these phases possess ferroelectric properties.

An analysis of Table 1 showed that there is no irrep that is included simultaneously in the permutation representations on both the $A$ and $B$ sites in the perovskite structure. This means that there cannot exist a single OP that describes the formation of a perovskite-like structure with simultaneous $A$ and $B$-site ordering. For this reason, in Fig. 1 and in the present work, $B$-site ordering is considered only in combinations with other OPs, but not as 'one order parameter'.

Many phases with the same structures can be obtained by different paths (Fig. 1). For example, the structure of the rhombohedral phase with space group $R \overline{3} m$ and $V / V_{0}=4$ can be induced by the tilts of octahedra or by the combination of $A$-site ordering and $X$-site ordering or by the combination of $A$-site ordering and $B$-site ordering etc. This complicates the analysis of similar phases in Table 2, where the proper and improper OPs, the primitive cell translations and the changes in their volumes, as well as structural formulas are given.
Therefore, in what follows, only some (possibly minimal) sets of proper OPs from the full set of OPs will be given for these phases.

\section{Structural mechanisms of 1:3 $A$-site-ordered perovskite formation}

\subsection{Proper ordering in the perovskite $A$ sublattice}

3.1.1. Case of one OP. There are no experimentally observed phase transitions from the parent phase with the cubic perovskite structure (archetype) to 1:3 $A$-site-ordered perovskite phases. Nevertheless, within the framework of the archetype concept, the genesis of various ordered phases from an archetype and the structural mechanism of their formation can be determined. Of course, such a structural mechanism is conditional. However, it plays a crucial role as it allows one to understand possible types of atom displacements and permutations that can cause the formation of the 1:3 $A$-siteordered perovskite phases from the archetype structure. Thus, by using the concept of structural mechanisms, the similarity, hierarchy and genesis of the studied crystal structures can be shown.

To list all possible low-symmetry phases with ordering in the $A$ sublattice, obtained from the archetype with the space group $P m \overline{3} m$, we consider the corresponding permutation representation on the $1 a$ Wyckoff position of the perovskite structure (Table 1). It contains the following set of irreps:

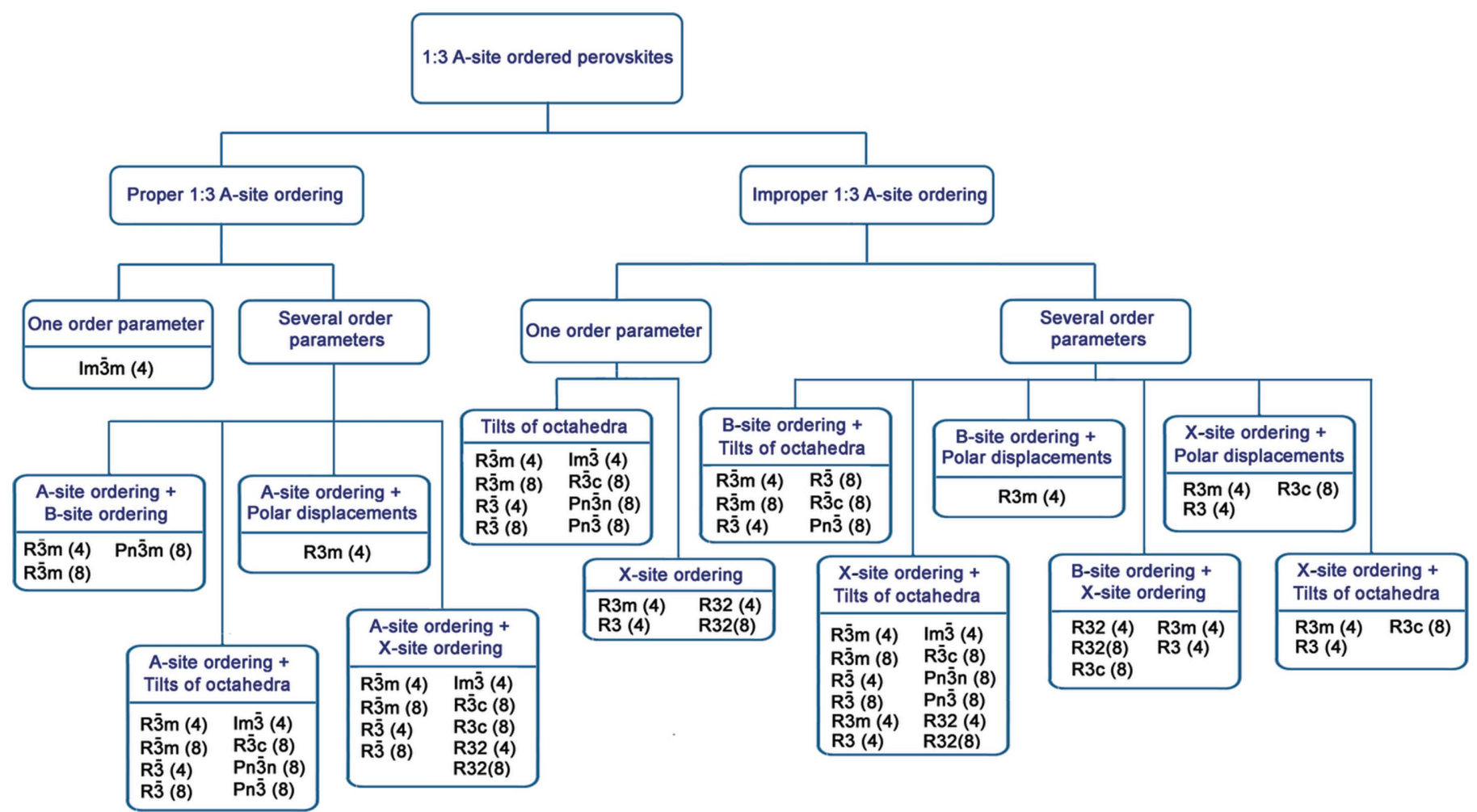

Figure 1

A classification of the OP combinations of 1:3 $A$-site-ordered perovskites that are considered in this work. The change in the primitive cell volume of the low-symmetry phase relative to the parent perovskite structure with the $P m \overline{3} m$ space group is indicated in brackets after the designation of the space group. 
Table 2

Possible low-symmetry 1:3 $A$-site-ordered perovskite phases characterized by different types of OPs.

The following designations of OPs are used: $\mathbf{k}_{10}(X)=\eta, \mathbf{k}_{11}(M)=\varphi, \mathbf{k}_{12}(\Gamma)=\sigma$ and $\mathbf{k}_{13}(R)=\Psi$. In the second and third columns, the directions of OPs in OP space are given. Then notations of the irreps according to Kovalev (1993) are provided. The designations of the irreps by Miller \& Love (1967) used in the ISOTROPY program are given in parentheses. The symbols $V, O, P$ and $D$ denote the physical nature of OPs associated with tilts of anion octahedra (or tilt-like distortions), atom orderings, and polar and non-polar atom displacements, respectively. The type of perovskite sublattice in which the OP is implemented is indicated by a subscript. The unit irrep $\mathbf{k}_{12}\left(\tau_{1}\right)\left(\Gamma_{1}^{+}\right)$is not shown. $V / V_{0}$ is the multiplication of the primitive cell volume as a result of virtual structural phase transition from the archetype structure. The superscript index in the structural formula means the type of Wyckoff position according to the International Tables for Crystallography.

\begin{tabular}{|c|c|c|c|c|}
\hline No. Proper OP & Improper OP & $\begin{array}{l}\text { Space } \\
\text { group }\end{array}$ & $\begin{array}{l}\text { Subgroup } \\
\text { basis/origin }\end{array}$ & $\begin{array}{l}\text { Structural } \\
V / V_{0} \text { formula }\end{array}$ \\
\hline $\begin{array}{l}\text { Proper 1:3 A-site ordering } \\
1 \quad(\varphi \varphi \varphi) ; \mathbf{k}_{11} \tau_{1}\left(M_{1}^{+}\right) ; O_{A} D_{X}\end{array}$ & & $\begin{array}{l}\operatorname{Im} \overline{3} m \\
\quad(\text { No. 229) }\end{array}$ & $\begin{array}{l}2 \mathbf{a}_{1}, 2 \mathbf{a}_{2}, 2 \mathbf{a}_{3} \\
\quad(0,0,0)\end{array}$ & $\begin{array}{c}A^{2 a}{ }_{1 / 4} A^{6 b}{ }_{3 / 4} \\
B^{8 c} \\
X^{24 h}{ }_{3}\end{array}$ \\
\hline
\end{tabular}

\section{$\boldsymbol{A}+\boldsymbol{B}$-site ordering}

$2(\varphi \varphi \varphi) ; \mathbf{k}_{11} \tau_{1}\left(M_{1}^{+}\right) ; O_{A} D_{X}$ $\Psi ; \mathbf{k}_{13} \tau_{4}\left(R_{2}{ }^{-}\right) ; O_{B} D_{X}$

$\boldsymbol{X}$-site ordering

$3(\varphi-\varphi \varphi-\varphi \varphi-\varphi) ; \mathbf{k}_{11} \tau_{10}\left(M_{5}^{-}\right)$; $O_{X} D_{A} D_{B} D_{X}$

$4 \quad(\varphi-\varphi \varphi-\varphi \varphi-\varphi) ; \mathbf{k}_{11} \tau_{10}\left(M_{5}{ }^{-}\right) ;$ $O_{X} D_{A} D_{B} D_{X}$ $(\eta \eta \eta) ; \mathbf{k}_{10} \tau_{4}\left(X_{3}^{-}\right)$; $O_{B} O_{X} D_{A} D_{X}$

Tilts of anion octahedra or tilt-like distortions

$5 \quad(\varphi \varphi \varphi) ; \mathbf{k}_{11} \tau_{5}\left(M_{2}^{+}\right) ; V_{B} D_{X}$

$6 \quad(\eta \eta \eta) ; \mathbf{k}_{10} \tau_{2}\left(X_{1}^{-}\right) ; V_{B}$

$7 \quad(\varphi-\varphi \varphi-\varphi \varphi-\varphi) ; \mathbf{k}_{11} \tau_{9}\left(M_{5}^{+}\right) ; V_{B} D_{X}$ or

$(\varphi \varphi \varphi) ; \mathbf{k}_{11} \tau_{1}\left(M_{1}^{+}\right) ; O_{A} D_{X}$ $(\varphi \varphi \varphi) ; \mathbf{k}_{11} \tau_{7}\left(M_{4}^{+}\right) ; O_{B} O_{X} D_{X}$

$8 \quad(\eta \eta \eta \eta \eta \eta) ; \mathbf{k}_{10} \tau_{10}\left(X_{5}^{-}\right) ; V_{B} D_{A} D_{X}$ or

$(\varphi \varphi \varphi) ; \mathbf{k}_{11} \tau_{1}\left(M_{1}^{+}\right) ; O_{A} D_{X}$ $(\eta \eta \eta) ; \mathbf{k}_{11} \tau_{4}\left(X_{3}^{-}\right) ; O_{B} D_{A} D_{X}$

$9 \quad\left(\varphi_{1} \varphi_{2} \varphi_{1} \varphi_{2} \varphi_{1} \varphi_{2}\right) ; \mathbf{k}_{11} \tau_{9}\left(M_{5}{ }^{+}\right) ; V_{B} D_{X}$ or

$(\varphi \varphi \varphi) ; \mathbf{k}_{11} \tau_{5}\left(M_{2}^{+}\right) ; V_{B} D_{X}$ $(\varphi \varphi \varphi) ; \mathbf{k}_{11} \tau_{7}\left(M_{4}^{+}\right) ; O_{B} O_{X} D_{X}$ $(\eta \eta \eta) ; \mathbf{k}_{13} \tau_{6}\left(X_{2}^{-}\right) ; D_{X}$

$\begin{array}{cl}P n \overline{3} m & -2 \mathbf{a}_{2},-2 \mathbf{a}_{1},-2 \mathbf{a}_{3} \quad 8 \\ (\text { No. 224) } & (3 / 2,3 / 2,3 / 2)\end{array}$

$A_{1 / 4}^{2 a} A_{3 / 4}^{6 d}$
$B^{4 b}{ }_{1 / 2} B^{4 c}{ }_{1 / 2}$
$X^{24 k}{ }_{3}$

$(\varphi \varphi \varphi) ; \mathbf{k}_{11} \tau_{1}\left(M_{1}^{+}\right) ; O_{A} D_{X} ;(\varphi \varphi \varphi)$
$\quad \mathbf{k}_{11} \tau_{2}\left(M_{1}^{-}\right) ;(\varphi \varphi \varphi) ; \mathbf{k}_{11} \tau_{7}\left(M_{4}^{+}\right) ;$

$O_{B} O_{X} D_{X} ;(\varphi \varphi \varphi) ; \mathbf{k}_{11} \tau_{8}\left(M_{4}^{-}\right) ;(\varphi-\varphi \varphi$

$-\varphi \varphi-\varphi) ; \mathbf{k}_{11} \tau_{9}\left(M_{5}^{+}\right) ; V_{B} D_{X} ; \sigma ;$

$\mathbf{k}_{12} \tau_{2}\left(\Gamma_{1}^{-}\right) ;(\sigma \sigma \sigma) ; \mathbf{k}_{12} \tau_{7}\left(\Gamma_{5}^{+}\right) ;(\sigma \sigma \sigma) ;$

$\mathbf{k}_{12} \tau_{8}\left(\Gamma_{5}{ }^{-}\right) ; D_{X}$

$(\eta \eta \eta) ; \mathbf{k}_{10} \tau_{5}\left(X_{2}^{+}\right) ;(\eta \eta \eta) ; \mathbf{k}_{10} \tau_{3}\left(X_{3}^{+}\right) ; O_{X} ;$

$(\eta \eta \eta \eta \eta \eta) ; \mathbf{k}_{10} \tau_{9}\left(X_{5}^{+}\right) ; D_{B} D_{X} ;(\eta \eta \eta)$;

$\mathbf{k}_{10} \tau_{6}\left(X_{2}^{-}\right) ; D_{X} ;(\eta \eta \eta \eta \eta \eta) ; \mathbf{k}_{10} \tau_{10}\left(X_{5}^{-}\right)$;

$V_{B} D_{A} D_{X} ;(\varphi \varphi \varphi) ; \mathbf{k}_{11} \tau_{1}\left(M_{1}^{+}\right) ; O_{A} D_{X} ;(\varphi$

$\varphi \varphi) ; \mathbf{k}_{11} \tau_{2}\left(M_{1}^{-}\right) ;(\varphi \varphi \varphi) ; \mathbf{k}_{11} \tau_{7}\left(M_{4}^{+}\right)$;

$O_{B} O_{X} D_{X} ;(\varphi \varphi \varphi) ; \mathbf{k}_{11} \tau_{8}\left(M_{4}^{-}\right) ;(\varphi-\varphi \varphi$

$-\varphi \varphi-\varphi) ; \mathbf{k}_{11} \tau_{9}\left(M_{5}^{+}\right) ; V_{B} D_{X} ; \sigma ;$

$\mathbf{k}_{12} \tau_{2}\left(\Gamma_{1}{ }^{-}\right) ;(\sigma \sigma \sigma) ; \mathbf{k}_{12} \tau_{8}\left(\Gamma_{5}{ }^{-}\right) ; D_{X} ;(\sigma \sigma$

$\sigma) ; \mathbf{k}_{12} \tau_{7}\left(\Gamma_{5}^{+}\right) ; \Psi ; \mathbf{k}_{13} \tau_{3}\left(R_{2}^{+}\right) ;(\Psi \Psi \Psi) ;$

$\mathbf{k}_{13} \tau_{9}\left(R_{4}{ }^{+}\right) ; \Psi ; \mathbf{k}_{13} \tau_{4}\left(R_{2}{ }^{-}\right) ; O_{B} D_{X} ;(\Psi \Psi$

$\Psi) ; \mathbf{k}_{13} \tau_{10}\left(R_{4}{ }^{-}\right) ; D_{A} D_{X}$

\begin{tabular}{|c|c|c|c|c|}
\hline$(\varphi \varphi \varphi) ; \mathbf{k}_{11} \tau_{1}\left(M_{1}^{+}\right) ; O_{A} D_{X} ; \sigma ; \mathbf{k}_{12} \tau_{3}\left(\Gamma_{2}^{+}\right)$ & $\begin{array}{l}\operatorname{Im} \overline{3} \\
\quad(\text { No. 204) }\end{array}$ & $\begin{array}{l}2 \mathbf{a}_{1}, 2 \mathbf{a}_{2}, 2 \mathbf{a}_{3} \\
\quad(0,0,0)\end{array}$ & 4 & $\begin{array}{c}A_{1 / 4}^{2 a} A^{6 b}{ }_{3 / 4}^{8 c} \\
B^{8 c}\end{array}$ \\
\hline$(\varphi \varphi \varphi) ; \mathbf{k}_{11} \tau_{1}\left(M_{1}^{+}\right) ; O_{A} D_{X} ; \Psi ; \mathbf{k}_{13} \tau_{2}\left(R_{1}^{-}\right)$ & $\begin{array}{l}\operatorname{Pn} \overline{3} n \\
\quad \text { (No. 222) }\end{array}$ & $\begin{array}{r}2 \mathbf{a}_{1}, 2 \mathbf{a}_{2}, 2 \mathbf{a}_{3} \\
\quad(1 / 2,3 / 2,3 / 2)\end{array}$ & 8 & $\begin{array}{c}A^{2 a}{ }_{1 / 4} A^{6 b}{ }_{3 / 4} \\
B^{8 c} \\
X^{24 h}\end{array}$ \\
\hline $\begin{array}{l}(\varphi \varphi \varphi) ; \mathbf{k}_{11} \tau_{1}\left(M_{1}^{+}\right) ; O_{A} D_{X} ;(\varphi \varphi \varphi) \\
\mathbf{k}_{11} \tau_{7}\left(M_{4}^{+}\right) ; O_{B} O_{X} D_{X} ;(\varphi-\varphi \varphi-\varphi \varphi-\varphi) \\
\mathbf{k}_{11} \tau_{9}\left(M_{5}^{+}\right) ; V_{B} D_{X} ;(\sigma \sigma \sigma) ; \mathbf{k}_{12} \tau_{7}\left(\Gamma_{5}^{+}\right)\end{array}$ & $\begin{array}{l}R \overline{3} m \\
\quad \text { (No. 166) }\end{array}$ & $\begin{array}{l}2 \mathbf{a}_{1}-2 \mathbf{a}_{3} \\
\quad-2 \mathbf{a}_{2}+2 \mathbf{a}_{3} \\
\quad-\mathbf{a}_{1}-\mathbf{a}_{2}-\mathbf{a}_{3} \\
(0,0,0)\end{array}$ & 4 & $\begin{array}{l}A_{1 / 4}^{3 a} A_{3 / 4}^{3} \\
B^{3 b}{ }_{1 / 4} B^{9 d} \\
X_{3 / 2}^{18 h} X_{3 / 2}^{18 f}\end{array}$ \\
\hline $\begin{array}{l}(\eta \eta \eta) ; \mathbf{k}_{10} \tau_{6}\left(X_{2}^{-}\right) ; D_{X} ;(\eta \eta \eta) ; \mathbf{k}_{10} \tau_{4}\left(X_{3}^{-}\right) \\
O_{B} O_{X} D_{A} D_{X} ;(\eta \eta \eta \eta \eta \eta) ; \mathbf{k}_{10} \tau_{10}\left(X_{5}^{-}\right) ; \\
V_{B} D_{A} D_{X} ;(\varphi \varphi \varphi) ; \mathbf{k}_{11} \tau_{1}\left(M_{1}^{+}\right) ; O_{A} D_{X} ;(\varphi \\
\varphi \varphi) ; \mathbf{k}_{11} \tau_{7}\left(M_{4}^{+}\right) ; O_{B} O_{X} D_{X} ;(\varphi-\varphi \varphi-\varphi \varphi \\
-\varphi) ; \mathbf{k}_{11} \tau_{9}\left(M_{5}^{+}\right) ; V_{B} D_{X} ;(\sigma \sigma \sigma) ; \\
\quad \mathbf{k}_{12} \tau_{7}\left(\Gamma_{5}^{+}\right) ; \Psi ; \mathbf{k}_{13} \tau_{4}\left(R_{2}{ }^{-}\right) ; O_{B} D_{X} ;(\Psi \Psi \\
\Psi) ; \mathbf{k}_{13} \tau_{10}\left(R_{4}^{-}\right) ; D_{A} D_{X}\end{array}$ & $\begin{array}{l}R \overline{3} m \\
\quad(\text { No. 166) }\end{array}$ & $\begin{array}{l}-2 \mathbf{a}_{1}+2 \mathbf{a}_{3} \\
\quad 2 \mathbf{a}_{2}-2 \mathbf{a}_{3} \\
-2 \mathbf{a}_{1}-2 \mathbf{a}_{2}-2 \mathbf{a}_{3} \\
(-1 / 2,-1 / 2,-1 / 2)\end{array}$ & 8 & $\begin{array}{l}A_{1 / 4}^{6 c} A^{18 h}{ }_{3 / 4} \\
\quad B_{1 / 8}^{3 a} B_{1 / 8}^{3 b} B_{3 / 8}^{9 d} B_{3 / 8}^{9 e} \\
\quad X_{3 / 2}^{18 h(2)} X_{3 / 2}^{36 i}\end{array}$ \\
\hline $\begin{array}{l}(\varphi \varphi \varphi) ; \mathbf{k}_{11} \tau_{1}\left(M_{1}^{+}\right) ; O_{A} D_{X} ;(\varphi \varphi \\
\varphi) ; \mathbf{k}_{11} \tau_{3}\left(M_{3}^{+}\right) ; D_{X} ;(\varphi \varphi \\
\varphi) ; \mathbf{k}_{11} \tau_{5}\left(M_{2}^{+}\right) ; V_{B} D_{X} ;(\varphi \varphi \\
\varphi) ; \mathbf{k}_{11} \tau_{7}\left(M_{4}^{+}\right) ; O_{B} O_{X} D_{X} ;\left(\varphi_{1} \varphi_{2} \varphi_{1} \varphi_{2} \varphi_{1}\right. \\
\left.\varphi_{2}\right) ; \mathbf{k}_{11} \tau_{9}\left(M_{5}^{+}\right) ; V_{B} D_{X} ; \sigma ; \mathbf{k}_{12} \tau_{3}\left(\Gamma_{2}{ }^{+}\right) ;(\sigma \sigma \\
\sigma) ; \mathbf{k}_{12} \tau_{7}\left(\Gamma_{5}^{+}\right) ;(\sigma \sigma \sigma) ; \mathbf{k}_{12} \tau_{9}\left(\Gamma_{4}^{+}\right) ; V_{B}\end{array}$ & $\begin{array}{l}R \overline{3} \\
\quad(\text { No. } 148)\end{array}$ & $\begin{array}{l}-2 \mathbf{a}_{1}+2 \mathbf{a}_{2} \\
-2 \mathbf{a}_{2}+2 \mathbf{a}_{3} \\
\mathbf{a}_{1}+\mathbf{a}_{2}+\mathbf{a}_{3} \\
(0,0,0)\end{array}$ & 4 & $\begin{array}{c}A^{3 a}{ }_{1 / 4} A^{9 e} e_{3 / 4} \\
B^{3 b}{ }_{1 / 4} B^{9 d}{ }_{3 / 4} \\
X^{18 f(2)}{ }_{3}\end{array}$ \\
\hline
\end{tabular}

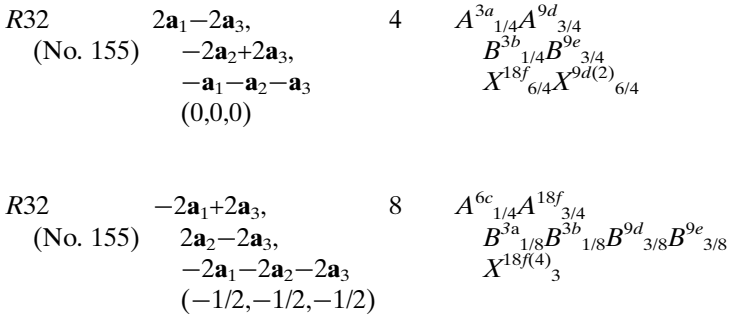
$232 \mathbf{a}_{1}-2 \mathbf{a}_{3}$,
$-2 \mathbf{a}_{2}+2 \mathbf{a}_{3}$
$-\mathbf{a}_{1}-\mathbf{a}_{2}-\mathbf{a}_{3}$
$4 \quad A^{3 a}{ }_{1 / 4} A^{9 d}{ }_{3 / 4}$ $B^{3 b}{ }^{1 / 4} B^{9 e}$
$X^{18 f}{ }_{6 / 4}^{3 / 4} X^{9 d(2)}$
$A^{6 c} A^{18 f}$ $B_{1 / 8}^{3 a} B_{1 / 8}^{3 b} B_{3 / 8}^{9 d} B_{3 / 8}^{9 e}$ $-2 \mathbf{a}_{1}-2 \mathbf{a}_{2}-2 \mathbf{a}_{3}$
$(-1 / 2,-1 / 2,-1 / 2)$

.


Table 2 (continued)

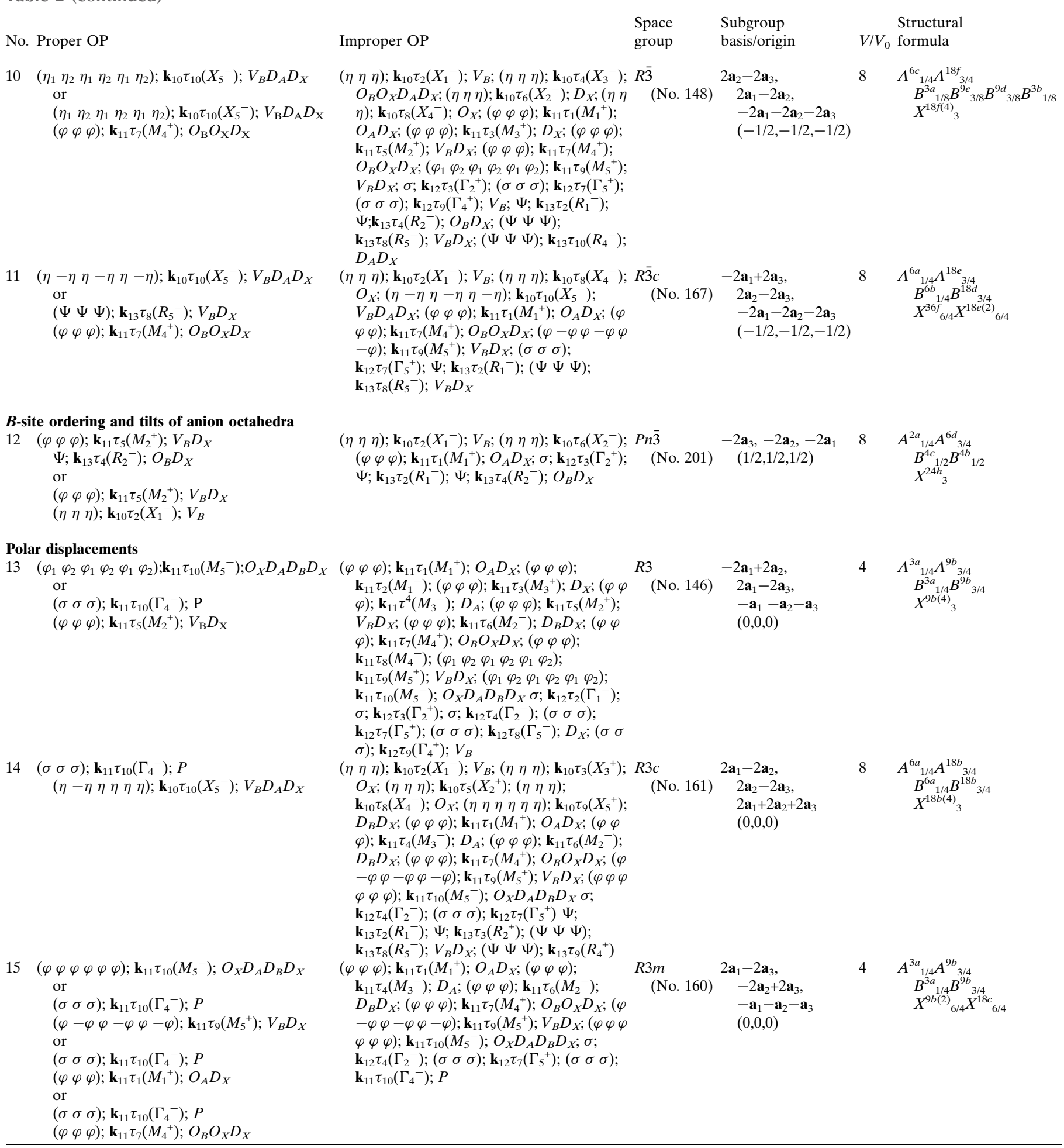

(Talanov, Talanov et al., 2014). However, only one of these phases with the $\operatorname{Im} \overline{3} m$ space group is characterized by $1: 3$ $A$-site ordering (Table 2). The three-component OP is transformed by the irrep $\mathbf{k}_{11} \tau_{1}\left(M_{1}{ }^{+}\right)$entering into the permutation representation (1). This irrep also enters into the mechanical representation on the $3 c$ Wyckoff position that leads to anion displacements in the ordered phase formation (Table 1). In this case, the structural formula of the ordered phase must be $A^{2 a}{ }_{1 / 4} A^{6 b}{ }_{3 / 4} B^{8 c} X^{24 h}$.

The given structure is an aristotype for a large number of derivatives, which can be obtained from this structure by $B$-site ordering, tilts of anion octahedra and other mechanisms. In this structure cation chains $A^{6 b}-A^{6 b}-A^{6 b}$ and $A^{6 b}-A^{2 a}$ $A^{6 b}$ are oriented in the directions [100], [010] and [001]. In the 


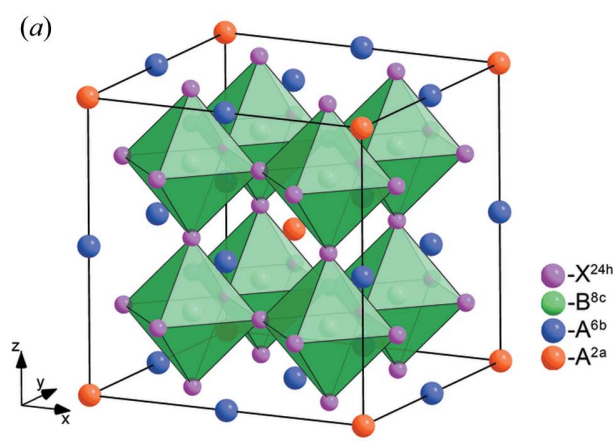

(b)

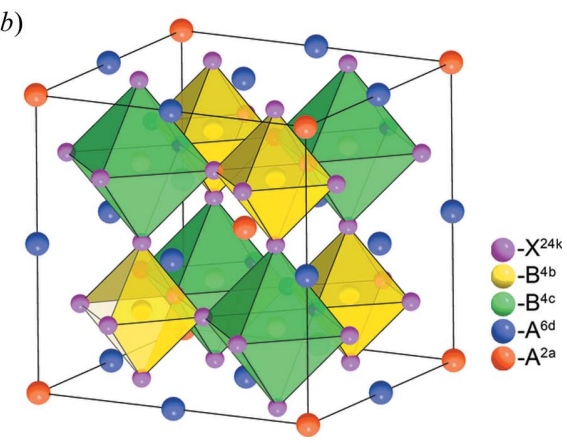

Figure 2

Calculated low-symmetry 1:3 $A$-site-ordered perovskite structures with space groups $(a) \operatorname{Im} \overline{3} m$ and (b) $\operatorname{Pn} \overline{3} m$.

$\left[B^{8 c} X^{24 h}{ }_{6}\right]$ octahedra, the $X^{24 h}$ atoms are located at the same distance from the central atom (Fig. $2 a$ ). The octahedra are linked to each other only by the vertices. There are not many substances with such a structure. This is explained by the fact that $B X_{6}$ octahedral distortions (in particular, tilts) are strongly dependent on the $A$-site cation size (Goldschmidt, 1926; Megaw \& Darlington, 1975; Thomas \& Beitollahi, 1994; Woodward, 1997b; Aso et al., 2014). As a result of distortions in the structure that arise due to dimensional mismatch of ions, additional OPs, in particular, the tilts of anion octahedra, will appear. Rare examples of the realization of this crystal structure are bismuth-containing superconducting oxides, $\left(\mathrm{Na}_{0.25} \mathrm{~K}_{0.45}\right)\left(\mathrm{Ba}_{1.00}\right)_{3}\left(\mathrm{Bi}_{1.00}\right)_{4} \mathrm{O}_{12}$, $\left(\mathrm{K}_{1.00}\right)\left(\mathrm{Ba}_{1.00}\right)_{3}\left(\mathrm{Bi}_{0.89} \mathrm{Na}_{0.11}\right)_{4} \mathrm{O}_{12}$ and $\mathrm{Ba}_{1-x} \mathrm{~K}_{x} \mathrm{Bi}_{1-y} \mathrm{Na}_{y} \mathrm{O}_{3}$, obtained by hydrothermal synthesis (Rubel et al., 2014, 2016; Zhang et al., 2011b).

3.1.2. Several OPs. (i) Combined proper atom ordering in $A$ and $B$ sublattices. The appearance of cation order in the $A$ sublattice can be associated with cation ordering in the $B$ sublattice. In this case, the transition to a low-symmetry structure is described by two independent OPs, which are transformed by the corresponding irreps entered into the permutation representation on the $1 a$ and $1 b$ Wyckoff positions of the perovskite structure. We obtained various lowsymmetry perovskite phases with simultaneous ordering in the $A$ and $B$ sublattices (Talanov, Shirokov et al., 2014). We showed that there exist 121 phases with simultaneous cation ordering in the $A$ and $B$ sublattices, and only three structures with 1:3 order in the $A$ sublattice: two $R \overline{3} m$ rhombohedral phases and one $P n \overline{3} m$ cubic phase (Table 2 ).

We consider in detail the structural mechanisms of $P n \overline{3} m$ phase formation. This phase is an aristotype of a large family of quadruple perovskites which are sometimes referred to as 1322 perovskites (Senn et al., 2014). ${ }^{\mathbf{5}}$ This structure is generated by two proper OPs that are transformed by 4D reducible representation, formed as a direct sum of irreps $\mathbf{k}_{11} \tau_{1}\left(M_{1}{ }^{+}\right)$ and $\mathbf{k}_{13} \tau_{4}\left(R_{2}{ }^{-}\right)$. Irrep $\mathbf{k}_{11} \tau_{1}\left(M_{1}^{+}\right)$enters into permutation representation on the $1 a$ Wyckoff position and also into

\footnotetext{
${ }^{5}$ From the aristotype under consideration, the structure of these perovskites is distinguished by the tilts of anion octahedra. The structural mechanisms of the formation of 1322 perovskites will be discussed in detail in Section 3.2.2.
}

mechanical representation on the $3 c$ Wyckoff position of the perovskite structure. Irrep $\mathbf{k}_{13} \tau_{4}\left(R_{2}{ }^{-}\right)$enters into permutation representation on the $1 b$ Wyckoff position and also into mechanical representation on the $3 c$ Wyckoff position. This means that lowsymmetry phase formation is accompanied by simultaneous $A$-cation and $B$-cation ordering and also by anion displacements. According to the grouptheoretical calculations the structural formula of the $P n \overline{3} m$ ordered perovskite is as follows: $A^{2 a}{ }_{1 / 4} A^{6 d}{ }_{3 / 4} B^{4 b}{ }_{1 / 2}-$ $B^{4 c}{ }_{1 / 2} X^{24 k}{ }_{3}$. The structure of this phase is shown in Fig. $2(b)$. In this structure, the distorted octahedra with the $B^{4 c}$ and $B^{4 b}$ central atoms are located in a checkerboard order. $A^{2 a}$ and $A^{6 d}$ cations in a ratio of 1:3 are ordered in voids formed by octahedra. The structure is characterized by an eightfold increase in the volume of the primitive cell. This theoretically predicted structure was found in loparite $\left(\mathrm{Na}_{0.59} \mathrm{Ce}_{0.41}\right)_{3}\left(\mathrm{Na}_{0.13} \mathrm{Ca}_{0.47} \mathrm{Ce}_{0.40}\right) \mathrm{Ti}_{2}\left(\mathrm{Ti}_{0.73} \mathrm{Nb}_{0.27}\right)_{2} \mathrm{O}_{12}$ (Zubkova et al., 2000). The distribution of atoms on Wyckoff positions in the structure of this mineral exactly corresponds to the theoretical result obtained: $A^{2 a}\left(\mathrm{Na}_{0.13} \mathrm{Ca}_{0.47} M^{*}\right)_{3} A^{6 d}$ $\left(\mathrm{Na}_{0.59} \mathrm{Ce}_{0.41}\right)_{3} \quad B^{4 c}\left(\mathrm{Ti}_{0.73} \mathrm{Nb}_{0.27}\right)_{2} B^{4 b}(\mathrm{Ti})_{1 / 2} X^{24 k}(\mathrm{O})_{12}$, where $M^{*}=\mathrm{Ce}_{0.19} \mathrm{La}_{0.08} \mathrm{Nd}_{0.04} \mathrm{Th}_{0.01} \mathrm{Sr}_{0.05} \operatorname{Pr}_{0.01} \mathrm{Eu}_{0.01} \mathrm{Sm}_{0.01}$.

(ii) Combined proper atom ordering in the A sublattice and polar displacements. The combined OP, which transforms according to the direct sum of the irreps $\mathbf{k}_{11} \tau_{1}\left(M_{1}^{+}\right)$and $\mathbf{k}_{12} \tau_{10}\left(\Gamma_{4}{ }^{-}\right)$, generates only one 1:3 $A$-site-ordered phase with the space group $R 3 m$ (more details in Section 3.2.2). The former irrep is responsible for the proper 1:3 $A$-site ordering, while the latter is responsible for the polar displacements. The structure of this phase is characterized by simultaneous atom ordering in all sublattices: 1:3 type of order in the $A$ and $B$ sublattices and 1:1:2 order type in the $X$ sublattice. The ordering in the $B$ and $X$ sublattices is related to the improper OPs transformed by irrep $\mathbf{k}_{11} \tau_{7}\left(M_{4}^{+}\right)$and by the direct sum of irreps $\mathbf{k}_{10} \tau_{4}\left(X_{3}{ }^{-}\right), \mathbf{k}_{11} \tau_{7}\left(M_{4}{ }^{+}\right), \mathbf{k}_{11} \tau_{10}\left(M_{5}{ }^{-}\right)$, respectively. Anion octahedra in the structure of this phase are distorted (nonGlazer tilts) due to the contribution of the OP $(\varphi-\varphi \varphi-\varphi \varphi$ $-\varphi$ ) transformed by irrep $\mathbf{k}_{11} \tau_{9}\left(M_{5}{ }^{+}\right)$. The polarization vector in this phase is directed along the solid diagonal [111]. This phase is similar to the structure generated by the OP associated with the ordering in the $X$ sublattice, but it is an improper ferroelectric, which is discussed in the next section.

\subsection{Improper ordering in the $A$ sublattice}

3.2.1. One OP. (i) Ordering of atoms in the $X$ sublattice. Atom ordering in the $A$ sublattice can be a secondary effect of atom ordering in other perovskite sublattices. We have shown previously that the ordering in the $B$ sublattice of the perovskite structure, described by a single OP, cannot lead to simultaneous ordering in the $A$ sublattice (Talanov, Talanov et al., 2014). Yet, the proposed ordering mechanism can be 
realized with anion ordering in the $X$ sublattice (Talanov et al., 2016). Of all the phases that are formed as a result of the anion ordering (induced by only one OP without the contribution of additional structural mechanisms), only three phases show 1:3 $A$-site ordering. These are rhombohedral phases with the space groups $R 3 m, R 3$ and $R 32$.

These phases are generated by one $6 \mathrm{D}$ irrep $\mathbf{k}_{11} \tau_{10}\left(M_{5}{ }^{-}\right)$, but characterized by different directions in OP space: $\left(\varphi_{1} \varphi_{2} \varphi_{1}\right.$ $\left.\varphi_{2} \varphi_{1} \varphi_{2}\right),(\varphi \varphi \varphi \varphi \varphi \varphi)$ and $(\varphi-\varphi \varphi-\varphi \varphi-\varphi)$. These phases have much in common:

(a) They are characterized by 1:3 $B$-site ordering, which is generated by improper irrep $\mathbf{k}_{11} \tau_{7}\left(M_{4}^{+}\right)$; this irrep enters into the permutation representations on the $1 b$ and $3 c$ Wyckoff positions of the perovskite structure [as well as in the mechanical representation on the $3 c$ Wyckoff position (Table 1)].

(b) They are characterized by quadruplications of the primitive volume cells relative to the parent perovskite structure.

(c) In the structures of these phases, tilt-like distortions generated by an improper OP transformed by irrep $\mathbf{k}_{11} \tau_{9}\left(M_{5}{ }^{+}\right)$ are possible. In the case of the $R 3$ phase the full set of the OPs also includes irreps $\mathbf{k}_{11} \tau_{5}\left(M_{2}^{+}\right)$and $\mathbf{k}_{12} \tau_{9}\left(\Gamma_{4}^{+}\right)$(Table 1).

(d) Irrep $\mathbf{k}_{11} \tau_{10}\left(M_{5}{ }^{-}\right)$also enters into mechanical representations on the $1 a$ and $1 b$ Wyckoff positions that describe the displacement of the $A$ and $B$ cations.

In addition, it is important to note that phases with $R 3 \mathrm{~m}$ and $R 3$ space groups are improper ferroelectrics (the structures of similar phases, which are proper ferroelectrics induced by the same full set of the OPs, are described in Section 3.1.2). All three phases are improper ferroelastics.

Besides, another phase with the $R 32$ space group and $V / V_{0}=$ 8 can be induced by two OPs transformed by the direct sum of irreps $\mathbf{k}_{11} \tau_{10}\left(M_{5}{ }^{-}\right)$and $\mathbf{k}_{10} \tau_{4}\left(X_{3}{ }^{-}\right)$(phase 4 of Table 2). The last irrep enters into the permutation representations on the $1 b$ Wyckoff position. The structure of this phase can also be attributed to the structures formed as a result of combined $X$ and $B$-site ordering (Fig. 1).

(ii) Tilts of anion octahedra. The tilts of anion octahedra along with the atom displacements or orderings can also be OPs. Note that the tilts of anion octahedra are the most common type of distortions in perovskites (Megaw, 1957). Glazer and Alexandrov obtained 23 systems of possible tilts of anion octahedra in perovskites and proposed designations for their description (Glazer, 1972, 1975; Aleksandrov, 1976). Based on the results of the group-theoretical analysis, it was found that the OPs associated with tilts of anion octahedra are transformed by two irreps: $\mathbf{k}_{11} \tau_{5}\left(M_{2}^{+}\right)$, in-phase rotation, and $\mathbf{k}_{13} \tau_{8}\left(R_{5}{ }^{-}\right)$, out-of-phase rotation. These OPs, as well as their combinations, initiate the formation of 14 types of structures with Glazer tilts of octahedra (Howard \& Stokes, 1998; Campbell et al., 2018).

In many studies, the superposition of the tilts of anion octahedra and other mechanisms of lattice distortions - atom displacements (Aleksandrov \& Bartolomé, 2001; Aleksandrov, 1978; Stokes et al., 2002; Torgashev et al., 2005), cation orderings (Howard et al., 2003, Howard \& Stokes, 2004;
Woodward, 1997a), Jahn-Teller distortions (Balachandran \& Rondinelli, 2013; Carpenter \& Howard, 2009; Howard \& Carpenter, 2010) - are considered. In the above works only 'simple' tilts of anion octahedra in-phase or out-of-phase were studied. However, as shown by Shirokov \& Torgashev (2004), the tilts of anion octahedra in the perovskite structure can be induced not by two, but by six OPs, transforming according to the following irreps:

$$
\begin{aligned}
& \mathbf{k}_{10} \tau_{2}\left(X_{1}^{-}\right)+\mathbf{k}_{10} \tau_{10}\left(X_{5}^{-}\right)+\mathbf{k}_{11} \tau_{5}\left(M_{2}^{+}\right)+\mathbf{k}_{11} \tau_{9}\left(M_{5}^{+}\right)+\mathbf{k}_{12} \tau_{9}\left(\Gamma_{4}^{+}\right) \\
& +\mathbf{k}_{13} \tau_{8}\left(R_{5}^{-}\right) .
\end{aligned}
$$

The absolute majority of perovskite-like structures with tilts of anion octahedra induced by one of the two OPs $\left[\mathbf{k}_{11} \tau_{5}\left(M_{2}^{+}\right)\right.$ and $\mathbf{k}_{13} \tau_{8}\left(R_{5}{ }^{-}\right)$] or a combination of them can be described by the Glazer tilt system. As noted by Howard \& Stokes (2005), distortions of structures generated by the 6D irreps $\mathbf{k}_{10} \tau_{10}\left(X_{5}^{-}\right)$and $\mathbf{k}_{11} \tau_{9}\left(M_{5}^{+}\right)$are not tilts of octahedra in Glazer approximations and, hence, cannot be described by the Glazer notation system. However, unconventional non-Glazer tilts are observed in hybrid organic-inorganic perovskites where additional degrees of freedom become possible (Duyker et al., 2016; Wu et al., 2018; Boström et al., 2018; Wei et al., 2018). That is why consideration of structures with non-Glazer tilts can be useful for describing possible types of distortions in a wide variety of compounds; therefore they are included in this section as 'tilt-like distortions'.

In order to solve the problem of enumerating all possible low-symmetry phases obtained from the parent phase of $\operatorname{Pm} \overline{3} m$ by the tilts of anion octahedra, we consider a fourcomponent (24D) OP. This is transformed by the direct sum of the above irreps. This OP can generate the formation of 156 low-symmetry phases. However, only eight phases are characterized by 1:3 $A$-site ordering (Fig. 1), none of the phases being improper ferroelectrics.

The formation of one phase $(\operatorname{Im} \overline{3})$ induced by the OP, transforming according to the irrep $\mathbf{k}_{11} \tau_{5}\left(\mathrm{M}_{2}^{+}\right)$, is accompanied by in-phase tilts of octahedra $\left(a^{+} a^{+} a^{+}\right.$Glazer tilt system) (Table 2). A phase with the $P n \overline{3} n$ space group is induced by the OP, transforming according to the irrep $\mathbf{k}_{10} \tau_{2}\left(X_{1}^{-}\right)$, which, in its turn, is associated with unconventional tilts of octahedra (Boström et al., 2018).

Each of the remaining six phases can be obtained as a result of a combination of different OPs: three phases induced by the OPs, transforming according to irrep $\mathbf{k}_{10} \tau_{10}\left(X_{5}^{-}\right)$, and two phases induced by the OPs, transforming according to irrep $\mathbf{k}_{11} \tau_{9}\left(M_{5}{ }^{+}\right)$. The former are formed with an eightfold increase in primitive cell volume, and the latter with a fourfold increase. In these structures, there is an ordering in the $B$ sublattice induced by improper OPs that are transformed by irreps $\mathbf{k}_{10} \tau_{4}\left(X_{3}{ }^{-}\right), \mathbf{k}_{11} \tau_{7}\left(M_{4}^{+}\right), \mathbf{k}_{13} \tau_{4}\left(R_{2}^{-}\right)$, which enter into the permutation representation of perovskite structure on the $1 b$ Wyckoff positions (Talanov et al., 2016). The $P n \overline{3}$ phase can be induced by a combination of several OPs only (see the next section).

We consider the $\operatorname{Im} \overline{\mathbf{3}}$ phase in more detail (phase 5 of Table 2, Fig. 3a) because there are a large number of 

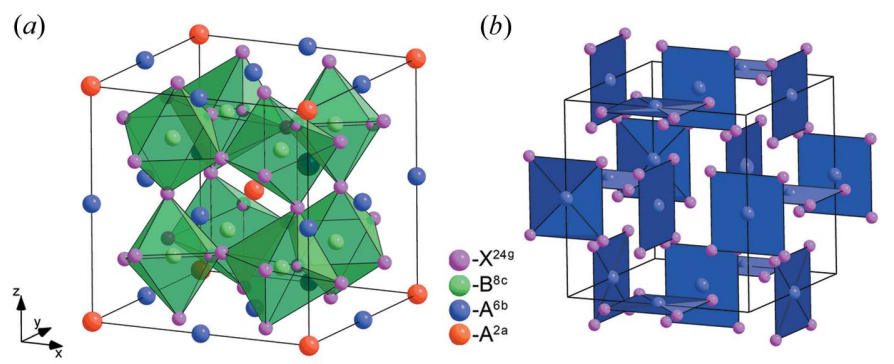

(c)
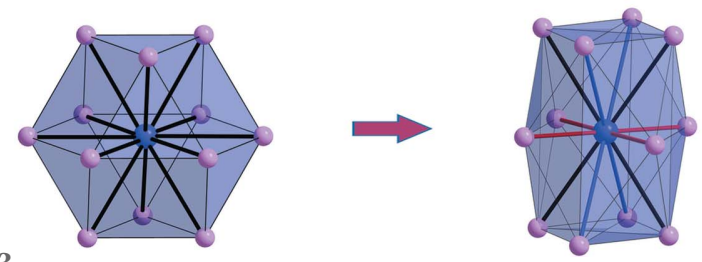

Figure 3

(a) Low-symmetry 1:3 $A$-site-ordered perovskite structures with space group $\operatorname{Im} \overline{3}$. (b) Square-planar coordination of $A^{6 b}$ cations. (c) The nearest environment of $A^{6 b}$ cations in $\operatorname{Im} \overline{3} m$ (left) and $\operatorname{Im} \overline{3}$ (right) structures. Short $A^{6 b}-X^{24 h / g}$ bonds are highlighted in red, medium in black, long in blue.

experimentally detected examples of such $1: 3 A$-site-ordered perovskites, in particular a family of $\mathrm{CaCu}_{3} \mathrm{Ti}_{4} \mathrm{O}_{12}$ materials (Subramanian et al., 2000; Belik, 2018). The structure of this phase is generated by a single OP $(\varphi \varphi \varphi)$, which is transformed by an irrep $\mathbf{k}_{11} \tau_{5}\left(M_{2}{ }^{+}\right)$. Two improper OPs $(\varphi \varphi \varphi)$ and $\sigma$, transforming according to irreps $\mathbf{k}_{11} \tau_{1}\left(M_{1}{ }^{+}\right)$and $\mathbf{k}_{12} \tau_{3}\left(\Gamma_{2}^{+}\right)$, respectively, are also included in the full set of OPs.

As a result of the in-phase tilts of anion octahedra with large inclination amplitude [in $\mathrm{CaCu}_{3} \mathrm{Ti}_{4} \mathrm{O}_{12}$ the $B-X-B$ bond angle is approximately equal to $140^{\circ}$ (Bochu et al., 1979)], a square-planar environment of $A^{6 b}$ cations arises (Fig. $3 b$ ). Thus, 12 identical distances $A-X$ (in the case of undistorted perovskite) are divided into four short, four medium and four long ones (Fig. 3c). This local configuration is stabilized by the Jahn-Teller effect in cases when the $A^{6 b}$ positions are occupied by $\mathrm{Cu}^{2+}$ or $\mathrm{Mn}^{3+}$ ions. Rare examples of $\operatorname{Im} \overline{\mathbf{3}}$ structure formation are the compounds with a substitution (total or partial) of Jahn-Teller ions on the non-JahnTeller ones: $\mathrm{Fe}^{2+}$ (Leinenweber et al., 1995), $\mathrm{Li}^{+}$(Mouron \& Choisnet, 1987), $\mathrm{Ti}^{4+}$ (Li et al., 2004; Avdeev \& Nalbandyan, 2006), $\mathrm{Co}^{2+}$ (Ovsyannikov, Zainulin et al., 2013), $\mathrm{Pd}^{2+}$ (Shiro et al., 2013) and $\mathrm{Pb}^{4+}$ (Sakai et al., 2017). In this case, a slight displacement of the $A^{6 b}$ atom in the direction perpendicular to the plane of square-planar oxygen coordination (Leinenweber et al., 1995; Ovsyannikov, Zainulin et al., 2013; Sakai et al., 2017) or splitting of $A^{6 b}$ positions (Tohyama et al., 2013; Shimura et al., 2016) is possible.

Relatively large $(\mathrm{Ca}, \mathrm{Sr}, \mathrm{Na}, \mathrm{Pb}, \mathrm{Bi})$ cations occupy the cubo-octahedral 12-coordinated $A^{2 a}$ positions. However, there are cases where smaller ions of transition metals $\mathrm{Mn}^{2+}$ (Akizuki et al., 2013) and $\mathrm{Cu}^{2+}$ (Akizuki et al., 2015) occupy these positions. In the $\mathrm{CuCu}_{3} \mathrm{~V}_{4} \mathrm{O}_{12}$ compound, there are significant temperature vibrations of $\mathrm{Cu}^{2+}$ ions in oversized cubo-octahedral cages, which are interpreted as a rattling effect (Jeitschko \& Braun, 1977). It is interesting to note the structural similarity of 1:3A-site-ordered perovskites with the $\operatorname{Im} \overline{3}$ space group to the widely studied class of cage compounds filled skutterudites $A^{2 a} B_{4}^{8 c} X_{12}{ }^{24 g}$ ( $A=$ rare-earth element, $B=$ transition metal, $X=$ pnicogen) with the same space group (Sales et al., 1996; Aleksandrov \& Beznosikov, 2007). Atoms $A$ rattle in the oversized $X_{12}$ icosahedral cage, reducing the lattice component of the thermal conductivity with a minor effect on the electronic properties (Sales et al., 1996; Nolas et al., 1999; Keppens et al., 1998). This feature allows filled skutterudites and other cage compounds to be considered as a promising class of thermoelectric materials.

The proximity of ion bond lengths of transition metals $A^{6 b}$ and $B^{8 c}$ with oxygen is another important peculiarity of $1: 3$ $A$-site-ordered perovskite structure with the $\operatorname{Im} \overline{3}$ space group. The above peculiarity appears as a result of tilts of octahedra (King \& Woodward, 2010). This suggests the possibility of electronic interactions through $A^{\prime}-X-B$ chains in addition to the interactions through $B-X-B$ chains that are characteristic of perovskites. As a result, new electronic effects, such as temperature-induced $A-B$ intersite charge transfer (Long, Hayashi et al., 2009; Long, Saito et al., 2009; Zhang et al., 2014; Long \& Shimakawa, 2010; Yamada et al., 2011, 2013, 2016; Chen et al., 2010) and a charge disproportionation (Yamada et al., 2008, 2013, 2016) are possible. Yamada et al. (2013), using the example of $\mathrm{LnCu}_{3} \mathrm{Fe}_{4} \mathrm{O}_{12}(\mathrm{Ln}=\mathrm{La}, \mathrm{Pr}, \mathrm{Nd}$, $\mathrm{Sm}, \mathrm{Eu}, \mathrm{Gd}, \mathrm{Tb}$ ) perovskites, show that in the case of large $\mathrm{Ln}$ ions an intersite charge transfer $\left(3 \mathrm{Cu}^{2+}+4 \mathrm{Fe}^{3.75+} \rightarrow 3 \mathrm{Cu}^{3+}+\right.$ $\left.4 \mathrm{Fe}^{3+}\right)$ may arise. However, with smaller $\mathrm{Ln}$ ions $(\mathrm{Ln}=\mathrm{Dy}, \mathrm{Ho}$, $\mathrm{Er}, \mathrm{Tm} \mathrm{Yb}, \mathrm{Lu})$ charge disproportionation $\left(8 \mathrm{Fe}^{3.75+} \rightarrow 5 \mathrm{Fe}^{3+}+\right.$ $3 \mathrm{Fe}^{5+}$ ) below $\sim 250-260 \mathrm{~K}$ occurs.

From the crystal chemical point of view, the closest to the $\operatorname{Im} \overline{3}$ structure is the $\operatorname{Im} \overline{3} m$ one (Table 2, Fig. 2a), which may be represented as an aristotype of the 1:3 $A$-site-ordered perovskites. The structure of the $\operatorname{Im} \overline{3}$ phase differs from the structure of the aristotype in anion positions: the anions in the structure of the $\operatorname{Im} \overline{3}$ phase occupy the two-parameter $24 \mathrm{~g}$ Wyckoff position with $m$ local symmetry, while in the $\operatorname{Im} \overline{3} m$ structure the anions occupy the one-parameter $24 h$ Wyckoff position with $m 2$ local symmetry. Thus, the $\operatorname{Im} \overline{3}$ structure can be represented as the $\operatorname{Im} \overline{3} m$ one with the in-phase tilts of anion octahedra characterized by the $(\varphi \varphi \varphi)$ OP that is transformed in accordance with the irrep $\mathbf{k}_{11} \tau_{5}\left(M_{2}{ }^{+}\right)$.

3.2.2. Several OPs. (i) Combination of B-site ordering and tilts of anion octahedra. To obtain the list of all possible 1:3 $A$-site-ordered phases resulting from the combination of $B$-site ordering and tilts of anion octahedra, it is necessary to consider the OP which is transformed according to the direct sum of irreps entered into the permutation representation of perovskite structure on the $1 b$ Wyckoff position and in the enumeration (2). The permutation representation has the form (Table 1):

$$
\mathbf{k}_{10} \tau_{4}\left(X_{3}^{-}\right)+\mathbf{k}_{11} \tau_{7}\left(M_{4}^{+}\right)+\mathbf{k}_{12} \tau_{1}\left(\Gamma_{1}^{+}\right)+\mathbf{k}_{13} \tau_{4}\left(R_{2}^{-}\right) .
$$

This nine-component OP, transforming according to the direct sum of (2) and (3), generates 174 phases, with only six of them being characterized by 1:3 $A$-site ordering, $B$-site ordering and tilts of anion octahedra (Fig. 1). Five out of six 


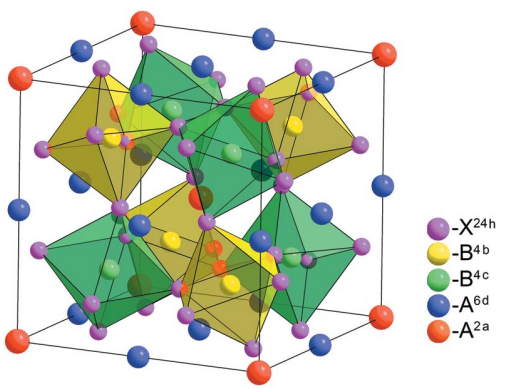

Figure 4

Calculated 1:3 $A$-site-ordered low-symmetry perovskite structures with space group $P n \overline{3}$.

resulting phases are induced by OPs, in the composition of which irrep $\mathbf{k}_{11} \tau_{7}\left(M_{4}{ }^{+}\right)$enters. This irrep is associated with 1:3 $B$-site ordering. This case can be seen in Table 2 (phases 7, 9 and 11). In the formation of phases 8 and 10, apart from the proper OP, improper OPs $\mathbf{k}_{13} \tau_{4}\left(R_{2}{ }^{-}\right)$and $\mathbf{k}_{10} \tau_{4}\left(X_{3}{ }^{-}\right)$participate. As a result, more complex 1:1:3:3 $B$-site ordering can be seen in these phases.

Let us consider in more detail the $P n \overline{3}$ phase formation mechanism (phase 12 of Table 2), since this phase is observed in a large number of quadruple perovskites, which are sometimes referred to as 1322 perovskites (Senn et al., 2014). This phase has a structure with eightfold primitive cell volume (Fig. 4). It is generated by a two-component OP, which is transformed by the direct sum of two irreps $\mathbf{k}_{11} \tau_{5}\left(M_{2}{ }^{+}\right)$and $\mathbf{k}_{13} \tau_{4}\left(R_{2}{ }^{-}\right)$[or $\mathbf{k}_{11} \tau_{5}\left(M_{2}{ }^{+}\right)$and $\mathbf{k}_{10} \tau_{2}\left(X_{1}{ }^{-}\right)$from the same full set of OPs], linked with the in-phase tilts of anion octahedra and 1:1 $B$-site ordering (rock-salt ordering type). 1:3 $A$-site ordering is generated by an improper OP $\mathbf{k}_{11} \tau_{1}\left(M_{1}{ }^{+}\right)$, which enters into the full set of OPs. This structure can be obtained in two ways from two different intermediate phases: from phase $\operatorname{Im} \overline{\overline{3}}$ by $1: 1 \quad B$-site ordering generated by the OP $\mathbf{k}_{13} \tau_{4}\left(R_{2}{ }^{-}\right)$or from phase $P n \overline{3} m$ by the tilts of anion octahedra induced by the OP $\mathbf{k}_{11} \tau_{5}\left(M_{2}^{+}\right)$. In the known cases, structural phase transitions in the $\operatorname{Pn} \overline{\mathbf{3}}$ phase pass through the first scenario and are accompanied by a charge disproportionation in the $B$ sublattice (Yamada et al., 2008, 2013, 2016; Meng et al., 2017).

The first synthesized compounds with simultaneous 1:3 ordering in the $A$ sublattice and 1:1 rock-salt ordering in (a)

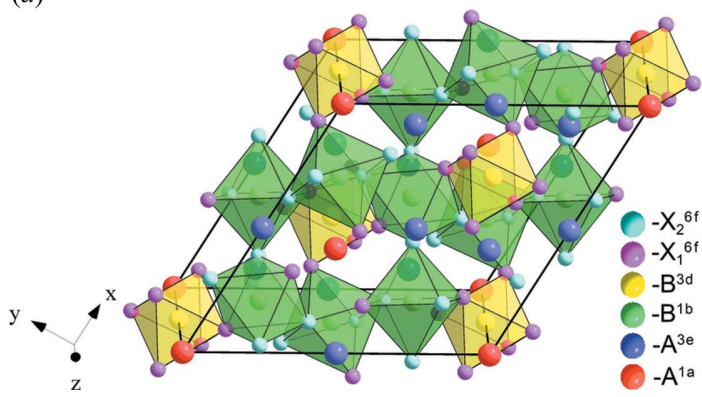

(b)

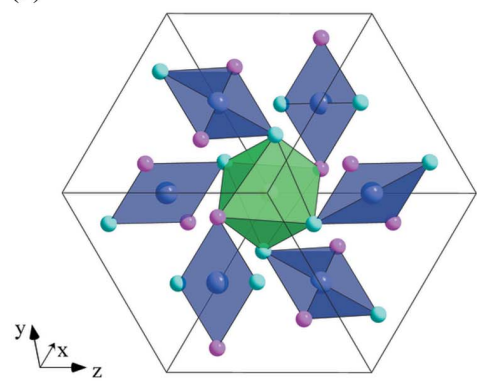

Figure 5

(a) Low-symmetry $A$-cation-ordered perovskite structure with space group $R \overline{3}$. (b) $R \overline{3}$ structure in the [111] direction. Only $A^{3 e}, B^{1 b}$ cations and anions are shown. Wyckoff positions are given for trigonal settings. the $B$ sublattice were $\mathrm{CaCu}_{3} \mathrm{Ga}_{2} \mathrm{Sn}_{2} \mathrm{O}_{12}$ and $\mathrm{CaCu}_{3} \mathrm{Ga}_{2} \mathrm{Ta}_{2} \mathrm{O}_{12}$ (Byeon et al., 2003). Later, a similar structure was discovered in $\mathrm{CaCu}_{3} \mathrm{Cr}_{2} \mathrm{Sb}_{2} \mathrm{O}_{12}$ (Byeon et al., 2005), $\mathrm{CaCu}_{3} \mathrm{Fe}_{2} \mathrm{Sb}_{2} \mathrm{O}_{12}$ (Chen et al., 2013) and $\mathrm{CaCu}_{3} \mathrm{Fe}_{2} \mathrm{Os}_{2} \mathrm{O}_{12}$ (Deng et al., 2016). The architecture of this structure allows the exchange interactions between $\mathrm{Cu}^{2+}$ and the magnetic $B$ cations to be influenced by introducing different cations in the $B^{\prime}$ sublattice. In addition, the nature of the magnetic properties depends on the degree of ordering in the $B$ sublattice, which, as shown by the example $\mathrm{CaCu}_{3} \mathrm{Fe}_{2} \mathrm{Nb}_{2} \mathrm{O}_{12}$, varies with different synthesis conditions (Senn et al., 2014). The compound $\mathrm{CaCu}_{3^{-}}$ $\mathrm{Fe}_{2} \mathrm{Re}_{2} \mathrm{O}_{12}$ is characterized by semi-metallic behavior with highly spin-polarized conduction electrons, a high Curie temperature and a significant magnetization, which are necessary for spintronic devices (Chen et al., 2014). Another unusual example of 1322 perovskites is $\mathrm{CaCu}_{3} \mathrm{Fe}_{4} \mathrm{O}_{12}$, a rare compound containing iron in higher oxidation states (Yamada et al., 2008). It demonstrates high catalytic activity and stability during oxygen evolution reaction $\left(4 \mathrm{OH}^{-} \rightarrow \mathrm{O}_{2}+2 \mathrm{H}_{2} \mathrm{O}+4 \mathrm{e}^{-}\right)$ (Yagi et al., 2015).

The calculated structure of phase $R \overline{3}$ (phase 9 of Table 2), which is observed in a rich group of ordered perovskites, is presented in Fig. 5(a). $\mathrm{CaMn}_{7} \mathrm{O}_{12}\left(\mathrm{CaMn}_{3} \mathrm{Mn}_{4} \mathrm{O}_{12}\right)$ (Bochu et al., 1980) is one of the compounds with such structure; it demonstrates a giant improper ferroelectricity as a result of the magnetic phase transition to an incommensurate helical magnetic structure below $90 \mathrm{~K}$ (Zhang et al., 2011a; Johnson et al., 2012; Perks et al., 2012). In the phase with multiferroic properties, the direction of the electric polarization axes, the direction of the magnetic helices and $\mathrm{MnO}_{6}$ rhombohedra twist coincide (Fig. 5b). According to Slawinski et al. (2009, 2010) in solid solutions $\mathrm{CaCu}_{x} \mathrm{Mn}_{7-x} \mathrm{O}_{12}$ at $x=0$ and 0.1 , modulation of magnetic moments and also atom displacements below $250 \mathrm{~K}$ can be seen.

The $R \overline{3}$ phase formation in $\mathrm{CaMn}_{7} \mathrm{O}_{12}$ occurs during the structural phase transition from the $\operatorname{Im} \overline{3}$ phase with cooling in the range $409-448 \mathrm{~K}$ and is accompanied by $1: 3$ charge ordering of $\mathrm{Mn}^{3+}$ and $\mathrm{Mn}^{4+}$ ions in the $B$ sublattice (Bochu et al., 1980; Przenioslo et al., 2002; Troyanchuk \& Chobot, 1997). From the point of view of the Landau theory of phase transitions, such a phase transition can be described by two OPs being transformed by irreps $\mathbf{k}_{11} \tau_{5}\left(M_{2}^{+}\right)$(in-phase tilts of anion octahedra) and $\mathbf{k}_{11} \tau_{7}\left(M_{4}^{+}\right)$(cation ordering in the $B$ sublattice) (Table 2) (Howard \& Stokes, 2004). However, a similar structure can be described by distortion of the $P m \overline{3} m$ parent structure generated by one OP transformed by irrep $\mathbf{k}_{11} \tau_{9}\left(M_{5}{ }^{+}\right)$from the same full set of OPs (Fig. 1, Table 2). The structural phase transition $\operatorname{Im} \overline{\mathbf{3}} \rightarrow R \overline{\mathbf{3}}$ was detected in other manganites: $A \mathrm{Mn}_{7} \mathrm{O}_{12}(A=\mathrm{Pb}$, $\mathrm{Sr}, \mathrm{Cd})$ (Glazkova et al., 2015; Belik, Glazkova, Terada et al., 2016). It is interesting to note that in $\mathrm{SrMn}_{7} \mathrm{O}_{12}$ (as well as $\left.\mathrm{CaMn}_{7} \mathrm{O}_{12}\right)$ structural modulations occur below $265 \mathrm{~K}[\mathbf{q}=(0,0$, 
0.9215 ) at $113 \mathrm{~K}$ ] (Belik, Glazkova \& Katsuyaal, 2016). In the case of $\mathrm{CdMn}_{7} \mathrm{O}_{12}$ and $\mathrm{PbMn}_{7} \mathrm{O}_{12}$, lowering of the temperature leads to another structural phase transition to commensurate $P \overline{3}$ phases at $T_{\mathrm{c}}=254 \mathrm{~K}$ and $294 \mathrm{~K}$, respectively (Belik, Glazkova \& Katsuyaal, 2016; Belik, Glazkova, Terada et al., 2016; Guo et al., 2017; Johnson et al., 2017). These phase transitions to the commensurate phases can be described by adding a third OP which condenses at the point (1/3 1/3 1/3) and is transformed by irrep $\mathbf{k}_{9} \tau_{1}$. A rare case of reentrant structural transitions $\operatorname{Im} \overline{\mathbf{3}} \rightarrow R \overline{\mathbf{3}} \rightarrow \operatorname{Im} \overline{3}$, which can be triggered by a magnetic field, was discovered in $\mathrm{BiCu}_{x} \mathrm{Mn}_{7-x} \mathrm{O}_{12}$ $(0.05 \leq x \leq 1.1)$, but its nature remains unknown (Belik, Matsushita \& Khalyavin, 2017).

An unusual form of the mixed metal oxalate $\mathrm{KLi}_{3} \mathrm{Fe}\left(\mathrm{C}_{2} \mathrm{O}_{4}\right)_{3}$ presented as a perovskite-like structure with 1:3 ordering of both $A$ and $B$ sites was reported by Yao et al. (2017). In this structure, the $\mathrm{Li}$ and $\mathrm{Fe}$ atoms are characterized by an octahedral oxygen environment, and the K atoms occupy only one fourth of the $A$ sites in the perovskite-like structure positions. Thus, the chemical formula of the compound in a perovskitelike form can be represented as $\mathrm{K}_{1 / 4} \square_{3 / 4}\left(\mathrm{Li}_{3 / 4} \mathrm{Fe}_{1 / 4}\right)\left(\mathrm{C}_{1.5} \mathrm{O}_{3}\right)$, where $\square$ is a vacancy. As noted by Yao et al. (2017), this structure is close to the calculated $R \overline{3} m$ structure generated by the combined atom ordering in $A$ and $B$ sublattices (phase 8 of Table 2). However, alternation of oxalate groups in the perovskite structure leads to distortion of anion octahedra and a symmetry change to the $R \overline{3} c$ space group (probably phase 11 of Table 2).

(ii) Combination of polar displacements and other mechanisms. In order to obtain a list of all possible 1:3 $A$-siteordered phases of proper ferroelectrics, it is necessary to choose an OP that transforms in the direct sum of the irrep $\mathbf{k}_{12} \tau_{10}\left(\Gamma_{4}{ }^{-}\right)$connected with the polarization, and the irreps responsible for the realization of other physical mechanisms: orderings in $A, B, X$ sublattices, and tilts of anion octahedra. All low-symmetry phases with 1:3 order in the $A$ sublattice, obtained by the above ways, are given in Table 2 and Fig. 1.

There are 457 phases induced by the combinations of polarization and tilts and anion octahedra: 391 phases by polarization and $X$-site ordering, 24 phases by polarization and $A$-site ordering. But there are only three different 1:3 $A$-site-ordered polar phases among them. The common factor
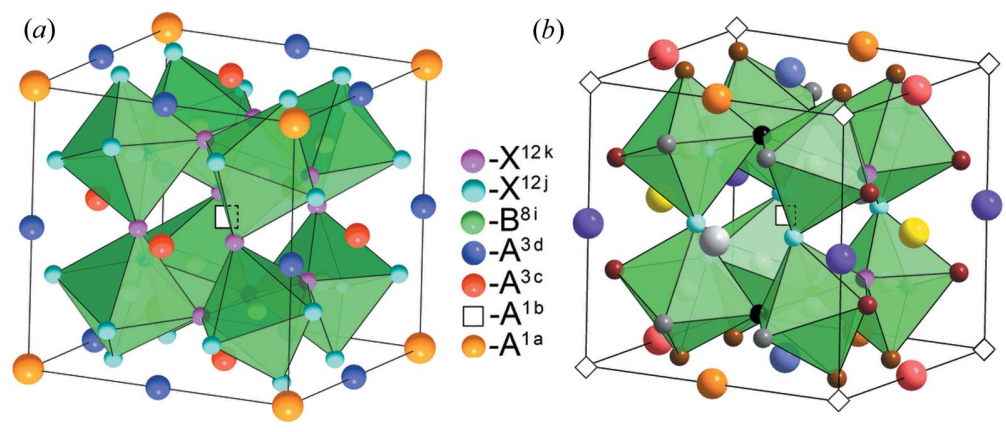

Figure 6

Calculated $A$-site-ordered low-symmetry perovskite structures with space group (a) $P m \overline{3}$ and (b) Pmmm.

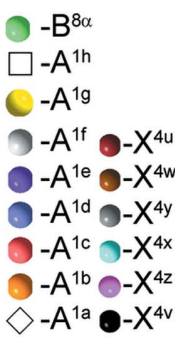

for all these ferroelectric phases is rhombohedral distortions of the crystal structures and 1:3 $B$-site ordering. The latter is related to the irrep $\mathbf{k}_{11} \tau_{7}\left(M_{4}^{+}\right)$entering into the full set of OPs for all phases given.

The phases with the $R 3 m$ space group (phase 15 of Table 2) are generated by OPs that are transformed by the direct sum of two irreps, one of which is $\mathbf{k}_{12} \tau_{10}\left(\Gamma_{4}{ }^{-}\right)$and the other $\mathbf{k}_{11} \tau_{1}\left(M_{1}{ }^{+}\right)$or $\mathbf{k}_{11} \tau_{10}\left(M_{5}{ }^{-}\right)$or $\mathbf{k}_{11} \tau_{7}\left(M_{4}{ }^{+}\right)$or $\mathbf{k}_{11} \tau_{9}\left(M_{5}{ }^{+}\right)$. They originate from the combinations of polarization with: proper ordering in the $A$ sublattice, ordering in the $X$ sublattice, ordering in the $B$ sublattice or the tilt-like distortion of the anion octahedra, respectively.

The phases with the space group $R 3$ (phases 13 of Table 2) are generated by the OP which is transformed by the direct sum of two irreps: one of them is $\mathbf{k}_{12} \tau_{10}\left(\Gamma_{4}{ }^{-}\right)$and the other is $\mathbf{k}_{11} \tau_{5}\left(M_{2}{ }^{+}\right)$or $\mathbf{k}_{11} \tau_{9}\left(M_{5}{ }^{+}\right)$or $\mathbf{k}_{11} \tau_{10}\left(M_{5}{ }^{-}\right.$) (Aleksandrov \& Bartolomé, 2001; Stokes et al., 2002; Torgashev et al., 2005). They originate from the combinations of polarization with $X$-site ordering or one of the two variants of octahedral tiltlike distortions, respectively. The structure of this phase is formed by proper or improper tilts of anion octahedra with rhombohedral displacements of $B$ atoms, co-directed with rotation axes (Stokes et al., 2002). The structure of this phase is observed in the quadruple perovskite $\mathrm{Bi}_{1-x / 3}\left(\mathrm{Mn}_{3}{ }_{3}\right)$ $\left(\mathrm{Mn}^{3+}{ }_{4-x} \mathrm{Mn}^{4+}{ }_{x}\right) \mathrm{O}_{12}$ in the range $0.10<x<0.27$ (Mezzadri et al., 2011).

The phase with the $R 3 c$ space group (phase 14 of Table 2) is generated by an OP that is transformed by the direct sum of two irreps $\mathbf{k}_{12} \tau_{10}\left(\Gamma_{4}{ }^{-}\right)$and $\mathbf{k}_{10} \tau_{10}\left(X_{5}{ }^{-}\right)$. The full set of the OPs of this phase includes 19 irreps, which reflect the complexity of this structure.

\subsection{Some particular cases of phase transitions in 1:3 ordered perovskites}

In this section we will consider the mechanisms of formation of some experimentally observed phases, which were not included in the general classification. The reason for this is either at phase transitions the type of ordering in the $A$ sublattice is more complicated than 1:3 (phases with space groups $P m \overline{3}, P m m m$ and $I 2 / m$ ), or the phase transition is associated with non-polar atom displacements (phases with space group I23).

3.3.1. $P m \overline{3}$ and $P m m m$ phase formation. Let us consider the $\mathrm{Ce}_{1 / 2} \mathrm{Cu}_{3} \mathrm{Ti}_{4} \mathrm{O}_{12}$ compound. Depending on the synthesis conditions, this compound exists in two crystalline forms: with random and ordered Ce/vacancy distribution at the $A$ site with $\operatorname{Im} \overline{\mathbf{3}}$ and $P m \overline{3}$ space groups, respectively (Saito et al., 2014) (Fig. 6a). $\mathrm{Ce} /$ vacancy ordering leads to the appearance of $L$-type ferrimagnetism instead of antiferromagnetism in the disordered structure. Another compound with this structure is $\mathrm{CuTa}_{2} \mathrm{O}_{6}$ (Propach, 1977), which can be 
(a)

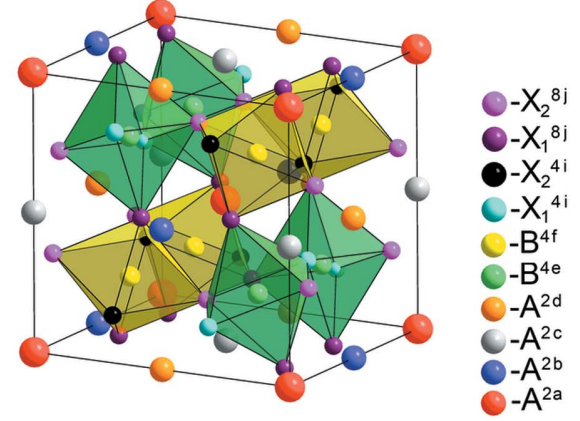

(b)

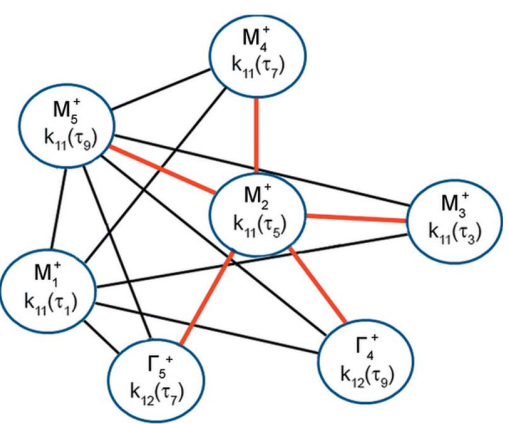

Figure 7

(a) Calculated $A$-cation-ordered low-symmetry perovskite structure with space group $I 2 / \mathrm{m}$. (b) Solid lines indicate pairs of irreps that induce the $12 / \mathrm{m}$ phase formation. Pairs that include $\mathbf{k}_{11} \tau_{5}\left(M_{2}^{+}\right)$are shown in red.

represented as a quadruple perovskite with a cationic deficit: $\square \mathrm{Cu}_{2} \square \mathrm{Ta}_{4} \mathrm{O}_{12}$.

The structural mechanisms responsible for the formation of an ordered modification with 1:1:3:3 order in the $A$ sublattice differ greatly from those for the disordered phase (Section 3.2.1). The phase structure $P m \overline{3}$ is generated by an OP that is transformed by the direct sum of two irreps: $\mathbf{k}_{11} \tau_{5}\left(M_{2}{ }^{+}\right)$and $\mathbf{k}_{13} \tau_{1}\left(R_{1}{ }^{+}\right)$. These irreps describe the tilts of anion octahedra (first irrep) and 1:1 $A$-site orderings (second irrep). The full set of the OPs also includes the irreps $\mathbf{k}_{11} \tau_{1}\left(M_{1}{ }^{+}\right)$(1:3 $A$-site ordering), $\mathbf{k}_{10} \tau_{1}\left(X_{1}^{+}\right)$( $A$-site ordering), $\mathbf{k}_{10} \tau_{5}\left(X_{2}^{+}\right), \mathbf{k}_{12} \tau_{3}\left(\Gamma_{2}^{+}\right)$ and $\mathbf{k}_{13} \tau_{3}\left(R_{2}{ }^{+}\right)$. The combinations of the irreps $\mathbf{k}_{12} \tau_{3}\left(\Gamma_{2}{ }^{+}\right)$and $\mathbf{k}_{10} \tau_{5}\left(X_{2}^{+}\right)$[or $\mathbf{k}_{10} \tau_{1}\left(X_{1}^{+}\right)$] from the full set of the OPs can act as the proper OPs. Note that the irrep $\mathbf{k}_{10} \tau_{1}\left(X_{1}{ }^{+}\right)$is connected with the 1:1:3:3 $A$-site ordering if the direction of the OP vector in OP space is $(\eta \eta \eta)$ (Talanov, Talanov et al., 2014).

According to Ebbinghaus (2007), the structure of slow-cooled samples of the nonstoichiometric compound $\mathrm{Cu}_{2+x} \mathrm{Ta}_{4} \mathrm{O}_{12+\sigma}$ corresponds to the Pmmm space group with the splitting of the $1 a$ Wyckoff position of the parent phase into eight single Wyckoff positions (only six of them are occupied by copper ions) (Fig. $6 b$ ). As in the case of the $P m \overline{3}$ phase, the structural phase transition to this phase can be described by an OP that is transformed by the direct sum of two irreps: $\mathbf{k}_{11} \tau_{5}\left(M_{2}{ }^{+}\right)$and $\mathbf{k}_{13} \tau_{1}\left(R_{1}{ }^{+}\right)$. But in the case of the Pmmm phase, the OP $\left(\varphi_{1} \varphi_{2} \varphi_{3}\right)$, generated by the irreps $\mathbf{k}_{11} \tau_{5}\left(\mathrm{M}_{2}{ }^{+}\right)$, corresponds to another system of tilts of anion octahedra: $a^{+} b^{+} c^{+}$, but not $a^{+} a^{+} a^{+}$(as in the case of the $P m \overline{3}$ phase) (Howard \& Stokes, 1998). As noted by Ebbinghaus (2007), the site occupancy of the six different copper sites can vary significantly, depending on the content of copper $(x)$ and the cooling speed (slow-cooling or quenching), giving rise to a cooperative vacancy ordering or to a statistical distribution.

3.3.2. I23 phase formation. The structure of $\mathrm{LiCuNb}_{3} \mathrm{O}_{9}$ [the structural formula is $\left(\mathrm{Li}_{8 / 3} \mathrm{Cu}_{8 / 3}\right)^{6 b} \mathrm{Nb}^{8 c}{ }_{8} \mathrm{O}^{24 f}{ }_{24}$ ] has the space group 123 (Sato \& Hama, 1993). In addition, an analogous phase is observed in $\mathrm{Fe}^{2+}$ - and $\mathrm{Ni}^{2+}$-modified $\mathrm{CaCu}_{3} \mathrm{Ti}_{4} \mathrm{O}_{12}$ (Moriyama et al., 2013). According to Moriyama et al. (2013), ferroelectric properties are observed in these solid solutions. Concentration phase transition from the centrosymmetric $\operatorname{Im} \overline{3}$ phase into the non-centrosymmetric $I 23$ phase is characterized by additional $B$ - and $X$-atom displace-

ments (Sato \& Hama, 1993; Moriyama et al., 2013). There are three irreps $\mathbf{k}_{10} \tau_{1}\left(X_{1}^{+}\right), \mathbf{k}_{10} \tau_{9}\left(X_{5}{ }^{+}\right)$and $\mathbf{k}_{11} \tau_{6}\left(M_{2}{ }^{-}\right)$ (Table 1) that enter into mechanical representation on the $1 b$ and $3 c$ Wyckoff positions of the archetype perovskite structure, but they do not enter into mechanical representation on the $1 a$ Wyckoff position (Table 1). However, only irrep $\mathbf{k}_{11} \tau_{6}\left(M_{2}{ }^{-}\right)$associated with $B$ - and $X$-site non-polar displacement and combined with irrep $\mathbf{k}_{11} \tau_{5}\left(M_{2}{ }^{+}\right)$induces the phase with the I23 space group. Improper OPs enter into the full set of OPs; they are transformed according to the following irreps: $\mathbf{k}_{12} \tau_{2}\left(\Gamma_{1}{ }^{-}\right)$, $\mathbf{k}_{12} \tau_{3}\left(\Gamma_{2}{ }^{+}\right), \mathbf{k}_{12} \tau_{4}\left(\Gamma_{2}{ }^{-}\right), \mathbf{k}_{11} \tau_{1}\left(M_{1}^{+}\right)$(1:3 $A$-site ordering) and $\mathbf{k}_{11} \tau_{2}\left(M_{1}{ }^{-}\right)$. It is important to note that irrep $\mathbf{k}_{12} \tau_{10}\left(\Gamma_{4}{ }^{-}\right)$, according to which the ferroelectric OP (the polarization) is transformed, does not enter into a full set of the OPs. This means that the structural phase transition from $\operatorname{Im} \overline{3}$ to the $I 23$ phase cannot be connected with the ferroelectric structural instabilities.

3.3.3. I $2 / m$ phase formation. In $\mathrm{LaMn}_{3} \mathrm{Mn}_{4} \mathrm{O}_{12}$, a phase transition from $I m \overline{3}$ to the $I 2 / m$ phase with a $1: 1 \mathrm{Mn}^{3+} / \mathrm{Mn}^{4+}$ charge ordering in the octahedral and 1:1:1 ordering in squareplanar positions is observed at $T_{\mathrm{c}}=653 \mathrm{~K}$ (Fig. 7a). It is accompanied by a change in the system of tilts of anion octahedra from $a^{+} a^{+} a^{+}$to $a^{+} b^{+} c^{+}$(Bochu et al., 1974; Okamoto et al., 2009). This phase is also observed in $R \mathrm{Mn}_{7} \mathrm{O}_{12}(R=\mathrm{Sm}$, $\mathrm{Eu}, \mathrm{Gd}$ and $\mathrm{Tb}$ ) (Zhang et al., 2018). In the case of potential multiferroic $\mathrm{BiMn}_{3} \mathrm{Mn}_{4} \mathrm{O}_{12}$ further cooling leads to $\mathrm{I} 2 / \mathrm{m} \rightarrow \mathrm{Im}$ $\rightarrow P 1$ phase transitions at $T=460 \mathrm{~K}$ and $290 \mathrm{~K}$, respectively (Imamura et al., 2008; Belik, Matsushita, Kumagai et al., 2017; Sławiński et al., 2017; Okamoto et al., 2010).

The phase transition $\operatorname{Im} \overline{\mathbf{3}} \rightarrow I 2 / m$ is described by a full set of OPs including the following irreps: $\mathbf{k}_{11} \tau_{1}\left(M_{1}{ }^{+}\right), \mathbf{k}_{11} \tau_{5}\left(M_{2}{ }^{+}\right)$, $\mathbf{k}_{11} \tau_{3}\left(M_{3}^{+}\right), \quad \mathbf{k}_{11} \tau_{7}\left(M_{4}^{+}\right), \quad \mathbf{k}_{11} \tau_{9}\left(M_{5}^{+}\right), \mathbf{k}_{12} \tau_{5}\left(\Gamma_{3}{ }^{+}\right), \quad \mathbf{k}_{12} \tau_{9}\left(\Gamma_{4}^{+}\right)$, $\mathbf{k}_{12} \tau_{7}\left(\Gamma_{5}{ }^{+}\right)$. There are 14 variants of irrep pair combinations, the direct sum of which forms a reducible representation, according to which the proper OP, which induces a lowtemperature phase, is transformed (Fig. $7 b$ ). Since it was previously shown that the phase $\operatorname{Im} \overline{3}$ is induced by the OP, which transforms by the irrep $\mathbf{k}_{11} \tau_{5}\left(M_{2}^{+}\right)$, it is necessary to consider the OP as one of the five combinations of irrep $\mathbf{k}_{11} \tau_{5}\left(M_{2}^{+}\right)$with irreps $\mathbf{k}_{12} \tau_{9}\left(\Gamma_{4}^{+}\right), \mathbf{k}_{12} \tau_{7}\left(\Gamma_{5}^{+}\right), \quad \mathbf{k}_{11} \tau_{3}\left(M_{3}^{+}\right)$, $\mathbf{k}_{11} \tau_{7}\left(M_{4}^{+}\right)$and $\mathbf{k}_{11} \tau_{9}\left(M_{5}{ }^{+}\right)$in order to describe the sequence of $P m \overline{3} m \rightarrow \operatorname{Im} \overline{3}$ (virtual phase transition) $\rightarrow I 2 / m$ phase transitions. Investigation of the temperature behavior of additional physical parameters, for example, the $C_{44}$ elastic modulus [the ferroelastic OP is transformed according to irrep $\mathbf{k}_{12} \tau_{7}\left(\Gamma_{5}^{+}\right)$, the degree of the tilts of anion octahedra $\left[\mathbf{k}_{11} \tau_{5}\left(M_{2}^{+}\right)\right.$and $\left.\mathbf{k}_{11} \tau_{9}\left(M_{5}^{+}\right)\right]$and others allows one to obtain more detailed information on the phase transition mechanisms.

The phase transition into the polar Im phase is described by a full set of the OPs, which also include OPs transforming by 


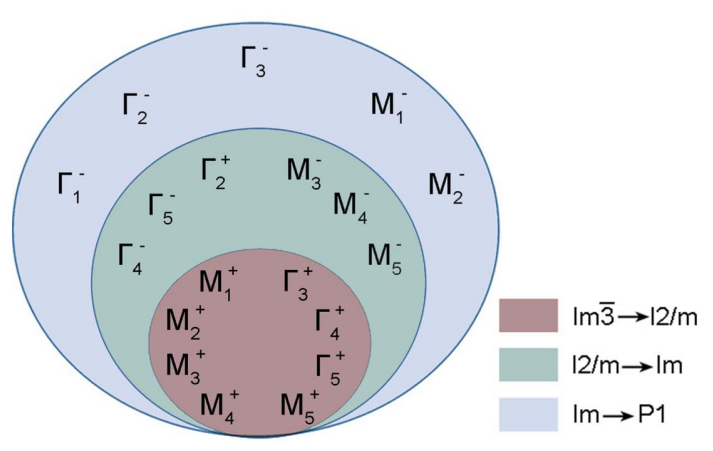

Figure 8

The full set of OPs for the description of the $\operatorname{Im} \overline{\mathbf{3}} \rightarrow I 2 / m \rightarrow \operatorname{Im} \rightarrow P 1$ phase transition cascade observed in $\mathrm{BiMn}_{3} \mathrm{Mn}_{4} \mathrm{O}_{12}$.

the irreps $\mathbf{k}_{12} \tau_{10}\left(\Gamma_{4}{ }^{-}\right), \mathbf{k}_{12} \tau_{8}\left(\Gamma_{5}{ }^{-}\right), \mathbf{k}_{12} \tau_{3}\left(\Gamma_{2}{ }^{+}\right), \mathbf{k}_{11} \tau_{4}\left(M_{3}{ }^{-}\right)$, $\mathbf{k}_{11} \tau_{8}\left(M_{4}{ }^{-}\right)$and $\mathbf{k}_{11} \tau_{10}\left(M_{5}{ }^{-}\right)$(Fig. 8). The entry into the full set of the OPs of the irrep $\mathbf{k}_{12} \tau_{10}\left(\Gamma_{4}{ }^{-}\right)$leads to the possibility of the appearance of ferroelectric properties in this phase. From the crystal chemical point of view, the stabilization of the polar phase is associated with the stereochemical effect induced by the presence of $\mathrm{Bi}^{3+}$ ions with the $6 s^{2}$ one pair of electrons (Belik, Matsushita, Kumagai et al., 2017; Sławiński et al., 2017; Mezzadri et al., 2009).

The phase transition to the triclinic $P 1$ phase is described by a full set of the OPs, which also includes OPs transforming by the irreps $\mathbf{k}_{12} \tau_{2}\left(\Gamma_{1}^{-}\right), \mathbf{k}_{12} \tau_{4}\left(\Gamma_{2}{ }^{-}\right), \mathbf{k}_{12} \tau_{6}\left(\Gamma_{3}{ }^{-}\right), \mathbf{k}_{11} \tau_{2}\left(M_{1}{ }^{-}\right)$and $\mathbf{k}_{11} \tau_{6}\left(M_{2}{ }^{-}\right)$(Fig. 8). The entry of irrep $\mathbf{k}_{11} \tau_{6}\left(M_{2}{ }^{-}\right)$into the full set of the OPs leads to additional $B$ - and $X$-atom displacements in the perovskite structure.

In another manganite $\mathrm{NaMn}_{7} \mathrm{O}_{12}\left(\mathrm{NaMn}_{3} \mathrm{Mn}_{4} \mathrm{O}_{12}\right)$ with $\mathrm{Mn}^{3+} / \mathrm{Mn}^{4+}$ charge ordering, an $\operatorname{Im} \overline{3} \rightarrow I 2 / m$ phase transition is observed in the temperature range 168-176 K (Prodi et al., 2004; Chenavas et al., 1975). However, further studies have shown that the commensurate modulation with a propagation vector $\mathbf{q}=(1 / 2,0,-1 / 2)$ can be seen in the low-temperature phase structure of this compound. Thus, the superstructure is described by the $C 2 / m$ space group (Prodi et al., 2014; Streltsov \& Khomskii, 2014).

\section{Conclusions}

Using group-theoretical methods of the phase transitions theory, possible 1:3 $A$-site-ordered structures are derived from more than 2600 low-symmetry perovskite-like phases obtained by different paths and then analyzed. These phases can be formed from a high-symmetry parent perovskite structure (archetype) as a result of real or virtual structural phase transitions connected with different OPs: atom orderings, atom displacements (polar and non-polar), tilts of octahedra and their combinations. From these phases all 1:3 $A$-site-ordered low-symmetry phases are identified and classified by irreps of the $\operatorname{Pm} \overline{3} m$ space group (Fig. 1, Table 2). Proper and improper OPs, as well as theoretically calculated structures (space groups, multiplications of primitive cells, Wyckoff position splitting) are presented for each of these phases. The structural mechanisms of the formation of some experimentally known 1:3 $A$-site-ordered low-symmetry phases from an archetype perovskite phase have been studied in detail: $\mathrm{CaCu}_{3} \mathrm{Ti}_{4} \mathrm{O}_{12}$ (the phase structure is characterized by the tilts of anion octahedra), $\mathrm{CaCu}_{3} \mathrm{Ga}_{2} \mathrm{Sn}_{2} \mathrm{O}_{12}$ (the phase structure can be represented by a combination of the tilts of anion octahedra and 1:1 $B$-site ordering), $\mathrm{CaMn}_{3} \mathrm{Mn}_{4} \mathrm{O}_{12}$ (the structure of the phase can be considered as a combination of the tilts of anion octahedra and 1:3 $B$-site ordering), $\mathrm{Ce}_{1 / 2^{-}}$ $\mathrm{Cu}_{3} \mathrm{Ti}_{4} \mathrm{O}_{12}$ (the phase structure is characterized by a combination of tilts of anion octahedra and additional 1:1 $A$-site ordering) and others.

Fig. 9 shows a modified Bärnighausen tree of all possible 1:3 $A$-site-ordered low-symmetry phases, based on the ITC approach (ITC, 2010; Müller, 2013) with the irreps indicated, describing relative phase transitions. Note the fundamental peculiarity of convergence of two approaches in the modified Bärnighausen tree. All group-subgroup relations when using the ITC approach are represented by different pairs of aristotype and hettotype phases.

In the case of the R-approach, irreps describe all the lowsymmetry phases that occur during a real or virtual phase transition from only one unchanged archetype phase, regardless of the intermediate phases. This is an important distinction. It significantly complicates the representation of the paths of formation of low-symmetry phases and the visualization of the results obtained. Therefore, the number of OPs describing the formation of some low-symmetry phases (Fig. 9) is not the minimum number of OPs. For example, from the modified Bärnighausen tree several OPs $\left[\mathbf{k}_{11} \tau_{1}\left(M_{1}^{+}\right)\right.$, $\left.\mathbf{k}_{11} \tau_{7}\left(M_{4}^{+}\right), \mathbf{k}_{13} \tau_{8}\left(R_{5}^{-}\right), \mathbf{k}_{10} \tau_{2}\left(X_{1}^{-}\right), \mathbf{k}_{11} \tau_{9}\left(M_{5}^{+}\right)\right]$can describe the formation of the phase with space group $R \overline{3} c$ (phase 11 , Table 2) depending on the path. In the case of the R-approach, the same phase can be obtained from the structure of the archetype by only one of its proper OPs, which are transformed by irrep $\mathbf{k}_{10} \tau_{10}\left(X_{5}{ }^{-}\right)$. However, the combination of the two approaches allows us to track the formation of a full set of OPs, which is unchanged for each of the phases regardless of the path, and fully determines its structure.

Note that all the phases obtained using the $\mathrm{R}$-approach are presented in Fig. 1 and Table 2, as well as Fig. 9. But in Fig. 9, there are several phases that were not obtained by using the $\mathrm{R}$-approach. These are phases with space groups $I \overline{4} 3 m, I 432$, $P m \overline{3} n, P \overline{4} 3 n$ and $P 4_{2} 32$. Let us explain this inconsistency in the results obtained by the R- and ITC approaches. The program COPL (Stokes \& Hatch, 2002; Hatch \& Stokes, 2002) helped us to ascertain that the first three phases are induced by OPs, which are transformed by irreps $\mathbf{k}_{11} \tau_{6}\left(M_{2}{ }^{-}\right)$, $\mathbf{k}_{11} \tau_{2}\left(M_{1}{ }^{-}\right)$and $\mathbf{k}_{10} \tau_{5}\left(X_{2}{ }^{+}\right)$, respectively. Irrep $\mathbf{k}_{11} \tau_{6}\left(M_{2}{ }^{-}\right)$ enters into mechanical representation on Wyckoff positions $1 b$ and $3 c$ of the perovskite structure (Table 1 ). This irrep induces non-polar displacements of $B$ and $X$ atoms and, therefore, it has not been considered. Irreps $\mathbf{k}_{11} \tau_{2}\left(M_{1}{ }^{-}\right)$and $\mathbf{k}_{10} \tau_{5}\left(X_{2}{ }^{+}\right)$ enter neither into permutational nor mechanical representations of the perovskite structure. According to Boström et al. (2018) OPs transforming by irreps $\mathbf{k}_{11} \tau_{6}\left(M_{2}{ }^{-}\right)$and $\mathbf{k}_{10} \tau_{5}\left(X_{2}{ }^{+}\right)$ are associated with columnar shifts and quadrupolar $A$-site order in molecular perovskites. The phases with space groups 
$P \overline{4} 3 n$ and $P 4_{2} 32$ can be induced by the combination of the OPs $\mathbf{k}_{12} \tau_{4}\left(\Gamma_{2}^{-}\right)$and $\mathbf{k}_{10} \tau_{5}\left(X_{2}^{+}\right)$in the first case, and $\mathbf{k}_{11} \tau_{2}\left(M_{1}{ }^{-}\right)$and $\mathbf{k}_{13} \tau_{3}\left(R_{2}^{+}\right)$or $\mathbf{k}_{12} \tau_{2}\left(\Gamma_{1}^{-}\right)$and $\mathbf{k}_{10} \tau_{5}\left(X_{2}^{+}\right)$in the second case. The physical nature of these parameters is unknown. The 1:3 $A$-site ordering in all five cases is explained by the occurrence of the improper OP transformed by irrep $\mathbf{k}_{11} \tau_{1}\left(M_{1}{ }^{+}\right)$in the full set of OPs. Thus, the five phases induced by the indicated irreps are beyond the limitations considered (Fig. 1). Besides, note the existence of two similar phases with the same space group $R \overline{3} c$, but with the different splittings of Wyckoff positions. Atom Wyckoff positions from the $A$ sublattice of the first phase correspond to atom Wyckoff positions from the $B$ sublattice of the second phase and vice versa. This is due to the internal automorphism of the positions $1 a$ and $1 b$ in the perovskite structure. Thus, the structure of one of the phases is induced by the OP transformed by irrep $\mathbf{k}_{13} \tau_{9}\left(R_{4}{ }^{+}\right)$, which is equivalent to the irrep $\mathbf{k}_{13} \tau_{8}\left(R_{5}{ }^{-}\right)$in the case when the origin is at the $1 b$ position. But when the origin is at the $1 a$ position, as in this work, the physical meaning of this OP is unclear, and that is why irrep $\mathbf{k}_{13} \tau_{9}\left(R_{4}^{+}\right)$has not been considered.

Fundamentally important is the following result. Most of the hettotype phases presented here can be obtained in various ways from different aristotype phases (Fig. 9). For example, the phase with space group $P n \overline{3}$ (hettotype) can be obtained from the aristotype phases $P n \overline{3} n, P n \overline{3} m$ or $\operatorname{Im} \overline{3}$ by $t 2$, by $t 2$ or by $k 2$, respectively, which, in turn, can be obtained from the phase with the space group $\operatorname{Im} \overline{3} m$ by $k 2$, by $k 2$ or by $t 2$, respectively (Figs. 9, 10). A similar situation arises in the

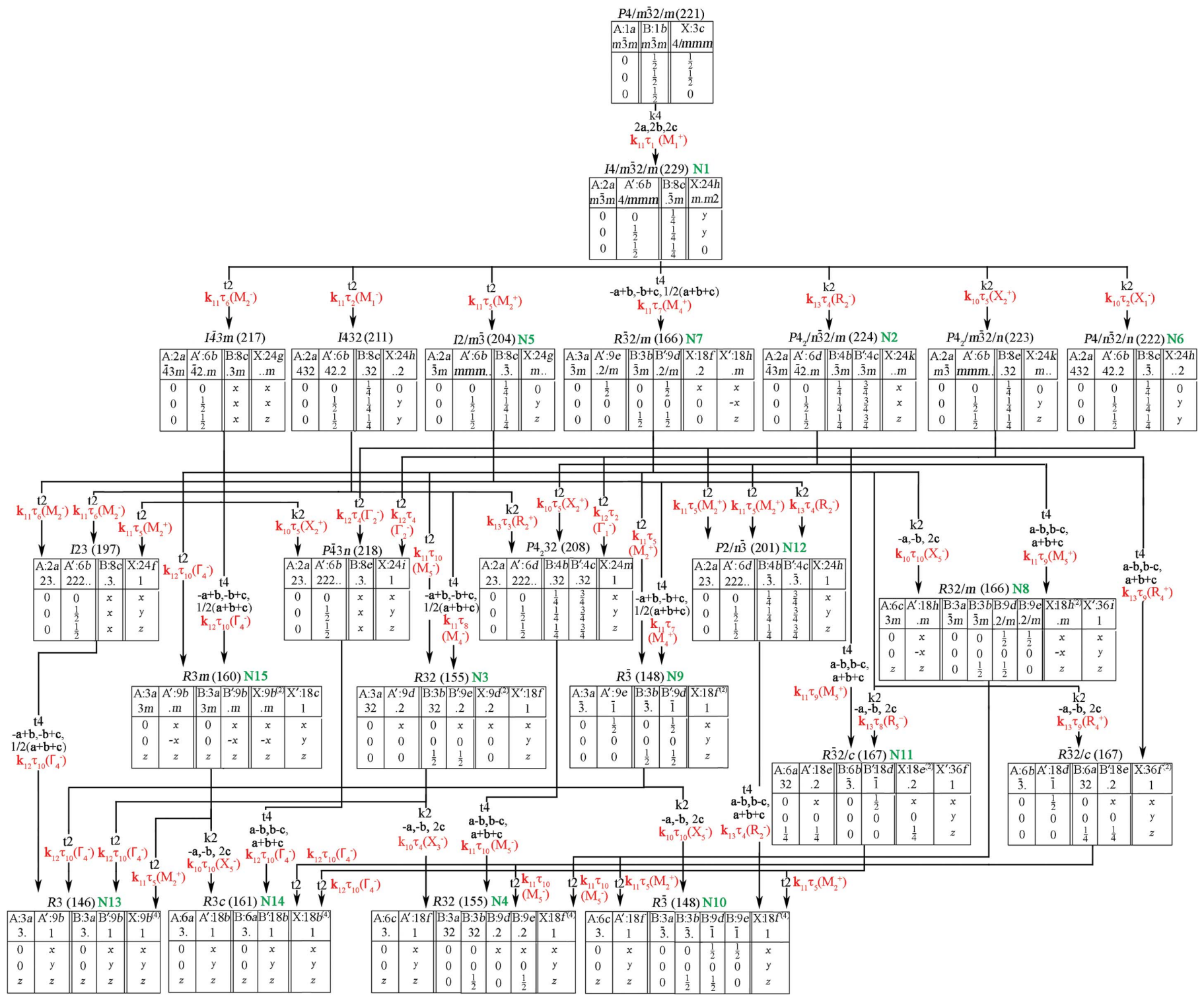

Figure 9

Modified Bärnighausen tree of all possible 1:3 $A$-site-ordered low-symmetry phases which was constructed using the ITC approach with the same limitations as in the R-approach $(2 a, 2 b, 2 c$ maximal primitive cell, only commensurate phases and 1:3 splitting type of $1 a$ Wyckoff position of archetype structure). The irreps are designated in red. The numbers of phases from Table 2 are designated in green. All translations of the primitive cells of the lowsymmetry phases in this figure relate to the primitive cell of the aristotype phase, which may vary for different phases of the hettotypes. In Table 2 , all translations are given relative to the primitive cell of a parent perovskite structure (archetype) that is common to all low-symmetry phases. 
variability found in selecting proper OPs from the full set of OPs in the R-approach (Fig. 1, Table 2). The same phase with the space group $P n \overline{3}$ can be induced from the phase with the space group $\operatorname{Im} \overline{3} m$ by three paths: in-phase tilts of octahedra [irrep $\mathbf{k}_{11} \tau_{5}\left(M_{2}{ }^{+}\right)$] and $B$-site ordering [irrep $\mathbf{k}_{13} \tau_{4}\left(R_{2}{ }^{-}\right)$] via the intermediate $\operatorname{Im} \overline{3}$ phase, or the reverse sequence of the same OPs via the intermediate $\operatorname{Pn} \overline{3} m$ phase or tilt-like distortions [irrep $\mathbf{k}_{10} \tau_{2}\left(X_{1}^{-}\right)$] and in-phase tilts of octahedra [irrep $\mathbf{k}_{11} \tau_{5}\left(M_{2}^{+}\right)$] via the intermediate $P n \overline{3} n$ phase. As stated above (Section 3.2.2), the known examples are realizations of the first scenario only (phase $P n \overline{3}$ is formed by $B$-site ordering). It should be noted that, regardless of the path of phase genesis, the full set of OPs remains unchanged.

The generalization of the result obtained is the principle of variation in selecting the paths for group-theoretical design of new phases. This principle can be formulated as follows: the same low-symmetry phase (hettotype) can be obtained from the high-symmetry phase (archetype) in several ways depending on the selection of the proper OPs from the full set of OPs, with the space-group type of the low-symmetry phase and the splitting of Wyckoff positions remaining the same, regardless of the genesis path of the low-symmetry phase.

An even more complicated case is observed during the description of the $\operatorname{Im} \overline{\mathbf{3}} \rightarrow I 2 / m \rightarrow \operatorname{Im} \rightarrow P 1$ phase transition cascade which occurs in $\mathrm{BiMn}_{3} \mathrm{Mn}_{4} \mathrm{O}_{12}$. At each step of the cascade, the number of irreps entering into a full set of the OPs increases. As a result, the number of options for choosing proper OPs in order to describe the entire sequence of structural phase transitions also increases. Thus, this is a first presentation of the variability in the choice of the proper OPs within the unchanged full set of OPs. This result emphasizes the need to consider the full set of OPs rather than proper OPs only. The observed variability in the choice of the proper OPs, which generate 1:3 $A$-site-ordered low-symmetry phases, highlights the fundamental significance of the full set of OPs when describing the structural phase transitions. The varia-

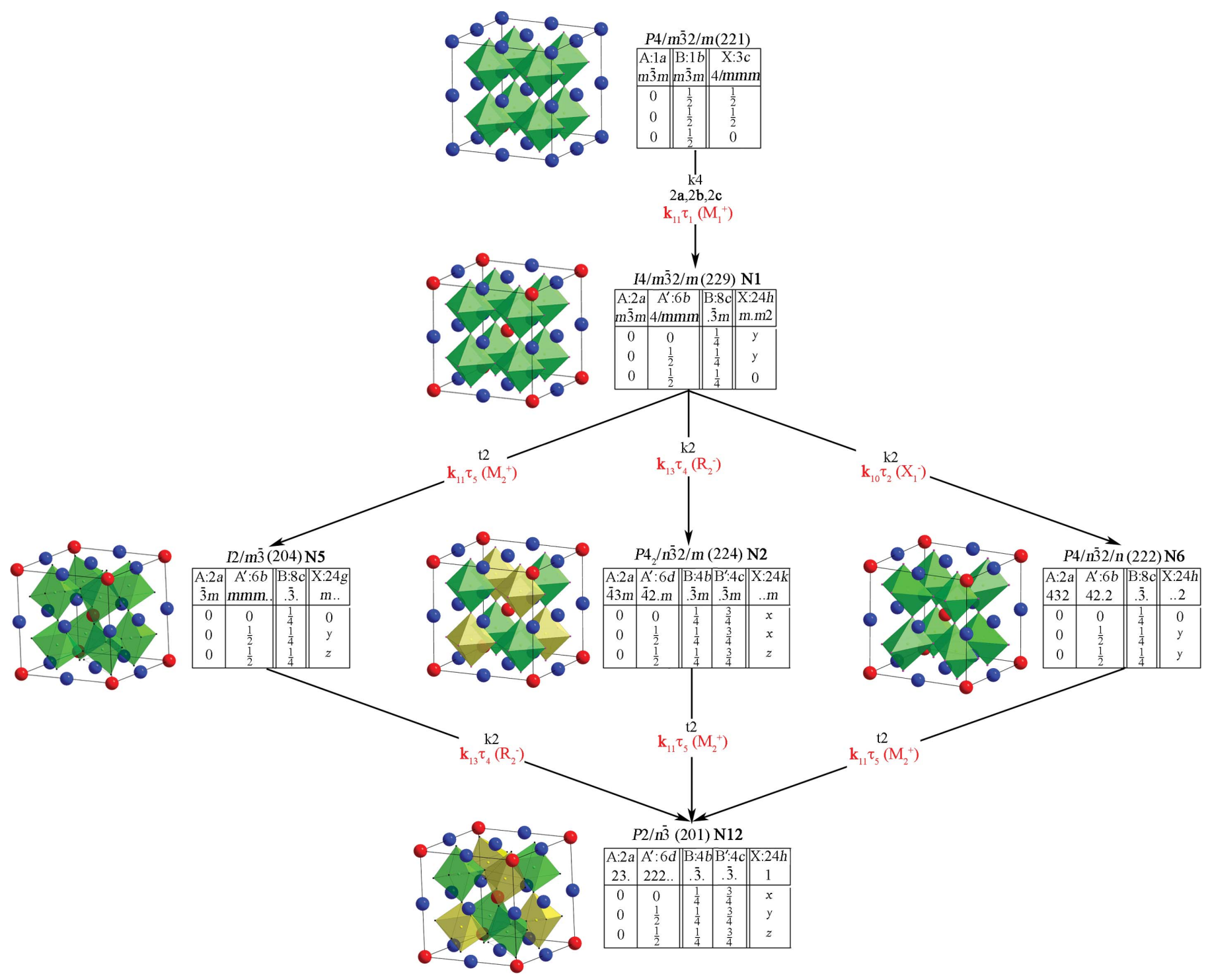

Figure 10

Possible group-theoretical paths for obtaining a phase with the space group $\operatorname{Pn} \overline{\mathbf{3}}$ by the ITC and R-approaches. 
bility in the choice of the proper OPs within the unchanged full set of OPs is a new phenomenon revealed in our study. This extraordinary and important phenomenon requires further research.

Thus, using the example of 1:3 $A$-site-ordered perovskites, this study showed that the combined use of ITC and $\mathrm{R}$-approaches allows determination of OPs that describe the structural phase transitions (including virtual transitions) and the structures of low-symmetry phases as well as investigation of the structural genesis and group-subgroup relations between different crystalline phases/structures.

\section{Acknowledgements}

The author is grateful to the referees for their constructive comments, for several useful discussions and their kind assistance in improving the clarity and readability of the manuscript. I express my sincere thanks to Professor Dr Ulrich Müller who introduced me to a very useful tool for theoretical analysis of group-subgroup relations (Bärnighausen formalism) and who helped in building the Bärnighausen tree. The author is also grateful to Professor Dr W. F. Kuhs for his kind interest, encouragement and constructive comments.

\section{Funding information}

The following funding is acknowledged: Russian Foundation for Basic Research (award No. 16-32-60025 mol_a_dk).

\section{References}

Adams, T. B., Sinclair, D. C. \& West, A. R. (2006). Phys. Rev. B, 73, 094124.

Akizuki, Y., Yamada, I., Fujita, K., Nishiyama, N., Irifune, T., Yajima, T., Kageyama, H. \& Tanaka, K. (2013). Inorg. Chem. 52, 1153811543.

Akizuki, Y., Yamada, I., Fujita, K., Taga, K., Kawakami, T., Mizumaki, M. \& Tanaka, K. (2015). Angew. Chem. Int. Ed. 54, 10870-10874.

Aleksandrov, K. S. (1976). Ferroelectrics, 14, 801-805.

Aleksandrov, K. S. (1978). Ferroelectrics, 20, 61-67.

Aleksandrov, K. S., Anistratov, A. T., Beznosikov, B. V. \& Fedoseeva, N. V. (1981). Phase Transitions in Crystal Halides $A B X_{3}$. Novosibirsk: Nauka.

Aleksandrov, K. S. \& Bartolomé, J. (2001). Phase Transit. 74, 255-335. Aleksandrov, K. S. \& Beznosikov, B. V. (2007). Crystallogr. Rep. 52, 28-36.

Aleksandrov, K. S. \& Beznosikov, V. V. (1997). Phys. Solid State, 39, 695-715.

Aroyo, M. I., Kirov, A., Capillas, C., Perez-Mato, J. M. \& Wondratschek, H. (2006). Acta Cryst. A62, 115-128.

Aso, R., Kan, D., Shimakawa, Y. \& Kurata, H. (2014). Cryst. Growth Des. 14, 2128-2132.

Avdeev, M. \& Nalbandyan, V. B. (2006). Inorg. Chem. 45, 2217-2220.

Balachandran, P. V. \& Rondinelli, J. M. (2013). Phys. Rev. B, 88, 054101.

Bärnighausen, H. (1975). Acta Cryst. A31, S3.

Bärnighausen, H. (1980). Commun. Math. Chem. 9, 139-175.

Belik, A. A. (2018). Dalton Trans. 47, 3209-3217.

Belik, A. A., Glazkova, Y. S., Katsuya, Y., Tanaka, M., Sobolev, A. V. \& Presniakov, I. A. (2016). Phys. Chem. C, 120, 8278-8288.
Belik, A. A., Glazkova, Y. S., Terada, N., Matsushita, Y., Sobolev, A. V., Presniakov, I. A., Tsujii, N., Nimori, S., Takehana, K. \& Imanaka, Y. (2016). Inorg. Chem. 55, 6169-6177.

Belik, A. A., Matsushita, Y. \& Khalyavin, D. D. (2017). Angew. Chem. Int. Ed. 56, 10423-10427.

Belik, A. A., Matsushita, Y., Kumagai, Yu., Katsuya, Y., Tanaka, M., Stefanovich, S. Yu., Lazoryak, B. I., Oba, F. \& Yamaura, K. (2017). Inorg. Chem. 56, 12272-12281.

Birman, J. L. (1978). Group-Theoretical Methods in Physics, edited by P. Kramers \& A. Reickers, pp. 203-222. Berlin: Springer Verlag.

Bochu, B., Buevoz, J. L., Chenavas, J., Collomb, A., Joubert, J. C. \& Marezio, M. (1980). Solid State Commun. 36, 133-138.

Bochu, B., Chenavas, J., Joubert, J. C. \& Marezio, M. (1974). J. Solid State Chem. 11, 88-93.

Bochu, B., Deschizeaux, M. N., Joubert, J. C., Collomb, A., Chenavas, J. \& Marezio, M. (1979). J. Solid State Chem. 29, 291-298.

Bock, O. \& Müller, U. (2002). Acta Cryst. B58, 594-606.

Boström, H. L. B., Senn, M. S. \& Goodwin, A. L. (2018). Nat. Commun. 9, 2380.

Bruce, A. D. \& Cowley, R. A. (1981). Structural Phase Transitions. London: Taylor and Francis.

Byeon, S.-H., Lee, S.-S., Parise, J. B., Woodward, P. M. \& Hur, N. H. (2005). Chem. Mater. 17, 3552-3557.

Byeon, S.-H., Lufaso, M. W., Parise, J. B., Woodward, P. M. \& Hansen, T. (2003). Chem. Mater. 15, 3798-3804.

Campbell, B., Howard, C. J., Averett, T. B., Whittle, T. A., Schmid, S., Machlus, S., Yost, C. \& Stokes, H. T. (2018). Acta Cryst. A74, 408424.

Campbell, B. J., Stokes, H. T., Tanner, D. E. \& Hatch, D. M. (2006). J. Appl. Cryst. 39, 607-614.

Carpenter, M. A. \& Howard, C. J. (2009). Acta Cryst. B65, 134-146.

Chechin, G. M. (1989). Comput. Math. Appl. 17, 255-278.

Chen, W., Mizumaki, M., Saito, T. \& Shimakawa, Y. (2013). Dalton Trans. 42, 10116-10120.

Chen, W., Mizumaki, M., Seki, H., Senn, M. S., Saito, T., Kan, D., Attfield, J. P. \& Shimakawa, Y. (2014). Nat. Commun. 5, 3909.

Chen, W.-T., Long, Y., Saito, T., Attfield, J. P. \& Shimakawa, Y. (2010). J. Mater. Chem. 20, 7282-7286.

Chenavas, J., Sayetat, F., Collomb, A., Joubert, J. C. \& Marezio, M. (1975). Solid State Commun. 16, 1129-1132.

Cohen, M. H., Neaton, J. B., He, L. \& Vanderbilt, D. (2003). J. Appl. Phys. 94, 3299-3306.

Deng, H., Liu, M., Dai, J., Hu, Z., Kuo, C., Yin, Y., Yang, J., Wang, X., Zhao, Q., Xu, Y., Fu, Z., Cai, J., Guo, H., Jin, K., Pi, T., Soo, Y., Zhou, G., Cheng, J., Chen, K., Ohresser, P., Yang, Y. F., Jin, C., Tjeng, L. H. \& Long, Y. (2016). Phys. Rev. B, 94, 024414.

Deschanvres, A., Raveau, B. \& Tollemer, F. (1967). Bull. Chim. Soc. Fr. pp. 4077-4078.

Dimmock, J. (1963). Phys. Rev. 130, 1337-1344.

Duyker, S. G., Hill, J. A., Howard, C. J. \& Goodwin, A. L. (2016). J. Am. Chem. Soc. 138, 11121-11123.

Ebbinghaus, S. G. (2007). Prog. Solid State Chem. 35, 421-431.

Fedorov, E. S. (1891). Simmetriia Pracil'nykh Sistem Figur. Zap. Min. Obshch. XXXVIII, 1.

Giovanoli, D. \& Leuenberger, U. (1969). Helv. Chim. Acta, 52, $2333-$ 2347.

Glazer, A. M. (1972). Acta Cryst. B28, 3384-3392.

Glazer, A. M. (1975). Acta Cryst. A31, 756-762.

Glazkova, Y. S., Terada, N., Matsushita, Y., Katsuya, Y., Tanaka, M., Sobolev, A. V., Presniakov, I. A. \& Belik, A. A. (2015). Inorg. Chem. 54, 9081-9091.

Goldschmidt, V. M. (1926). Naturwissenschaften, 14, 477-485.

Guo, H., Fernández-Díaz, M. T., Zhou, L., Yin, Y., Long, Y. \& Komarek, A. C. (2017). Sci. Rep. 7, 45939.

Hatch, D. M. \& Stokes, H. T. (2002). Phys. Rev. B, 65, 114113.

Hermann, C. (1928). Z. Kristallogr. 68, 257-287.

Homes, C. C., Vogt, T., Shapiro, S. M., Wakimoto, S. \& Ramirez, A. P. (2001). Science, 293, 673-676. 
Howard, C. J. \& Carpenter, M. A. (2010). Acta Cryst. B66, 40-50. Howard, C. J., Kennedy, B. J. \& Woodward, P. M. (2003). Acta Cryst. B59, 463-471.

Howard, C. J. \& Stokes, H. T. (1998). Acta Cryst. B54, 782-789.

Howard, C. J. \& Stokes, H. T. (2004). Acta Cryst. B60, 674-684.

Howard, C. J. \& Stokes, H. T. (2005). Acta Cryst. A61, 93-111.

Imamura, N., Karppinen, M., Motohashi, T., Fu, D., Itoh, M. \& Yamauchi, H. (2008). J. Am. Chem. Soc. 130, 14948-14949.

International Tables for Crystallography (2010). Vol. A1, Symmetry Relations between Space Groups, edited by H. Wondratschek \& U. Müller, 2nd ed. Chichester: John Wiley and Sons.

Jeitschko, W. \& Braun, D. (1977). Acta Cryst. B33, 3401-3406.

Johnson, R. D., Chapon, L. C., Khalyavin, D. D., Manuel, P., Radaelli, P. G. \& Martin, C. (2012). Phys. Rev. Lett. 108, 067201.

Johnson, R. D., Khalyavin, D. D., Manuel, P., Radaelli, P. G., Glazkova, I. S., Terada, N. \& Belik, A. A. (2017). Phys. Rev. B, 96, 054448.

Keppens, V., Mandrus, D., Sales, B. C., Chakoumakos, B. C., Dai, P., Coldea, R., Maple, M. B., Gajewski, D. A., Freeman, E. J. \& Bennington, S. (1998). Nature, 395, 876-878.

Kida, T., Kammuri, R., Hagiwara, M., Yoshii, S., Kobayashi, W., Iwakawa, M. \& Terasaki, I. (2012). Phys. Rev. B, 85, 195122.

King, G. \& Woodward, P. M. (2010). J. Mater. Chem. 20, 57855796.

Kobayashi, W., Terasaki, I., Takeya, J., Tsukada, I. \& Ando, Y. (2004). J. Phys. Soc. Jpn, 73, 2373-2376.

Kovalev, O. V. (1993). Representations of Crystallographic Space Groups. Irreducible Representations, Induced Representations and Co-representations. London: Taylor and Francis.

Landau, L. D. (1937). Zh. Eksp. Teor. Fiz. 7, 19-40.

Landau, L. D. \& Lifshitz, E. M. (1976). Course of Theoretical Physics, Vol. 5, Statistical Physics. Moscow: Nauka (Oxford: Pergamon, 1980).

Leinenweber, K., Linton, J., Navrotsky, A., Fei, Y. \& Parise, J. B. (1995). Phys. Chem. Miner. 22, 251-258.

Li, J., Subramanian, M. A., Rosenfeld, H. D., Jones, C. Y., Toby, B. H. \& Sleight, A. W. (2004). Chem. Mater. 16, 5223-5225.

Lifshitz, E. M. (1941). Zh. Eksp. Teor. Fiz. 11, 255-269.

Liu, Y., Withers, R. L. \& Wei, X. Y. (2005). Phys. Rev. B, 72, 134104. Long, Y. (2016). Chin. Phys. B, 25, 078108.

Long, Y., Saito, T., Tohyama, T., Oka, K., Azuma, M. \& Shimakawa, Y. (2009). Inorg. Chem. 48, 8489-8492.

Long, Y. \& Shimakawa, Y. (2010). New J. Phys. 12, 063029.

Long, Y. W., Hayashi, N., Saito, T., Azuma, M., Muranaka, S. \& Shimakawa, Y. (2009). Nature, 458, 60-63.

Lotgering, F. K. (1959). J. Inorg. Nucl. Chem. 9, 113-123.

Megaw, H. D. (1957). Ferroelectricity in Crystals. London: Methuen.

Megaw, H. D. (1973). Crystal Structures - a Working Approach. Philadelphia: W. B. Saunders.

Megaw, H. D. \& Darlington, C. N. W. (1975). Acta Cryst. A31, 161173.

Meng, J., Zhang, L., Yao, F., Zhang, X., Zhang, W., Liu, X., Meng, J. \& Zhang, H. (2017). Inorg. Chem. 56, 6371-6379.

Mezzadri, F., Buzzi, M., Pernechele, C., Calestani, G., Solzi, M., Migliori, A. \& Gilioli, E. (2011). Chem. Mater. 23, 3628-3635.

Mezzadri, F., Calestani, G., Calicchio, M., Gilioli, E., Bolzoni, F., Cabassi, R., Marezio, M. \& Migliori, A. (2009). Phys. Rev. B, 79, 100106.

Miller, S. C. \& Love, W. F. (1967). Tables of Irreducible Representations of Space Groups and Co-representations of Magnetic Space Groups. Boulder: Pruett.

Mitchel, R. H. (2002). Perovskites, Modern and Ancient. Canada: Almaz Press.

Molokeev, M. S. \& Misyul', S. V. (2012). Phys. Solid State, 54, 155-165.

Moriyama, T., Kan, A. \& Ogawa, H. (2013). Mater. Sci. Eng. B, 178, 875-880.

Mouron, P. \& Choisnet, J. (1987). J. Solid State Chem. 66, 311-317.

Müller, U. (2004). Z. Anorg. Allg. Chem. 630, 1519-1537.
Müller, U. (2013). Symmetry Relationships between Crystal Structures. Applications of Crystallographic Group Theory in Crystal Chemistry. Oxford University Press.

Nolas, G. S., Morelli, D. T. \& Tritt, T. M. (1999). Annu. Rev. Mater. Sci. 29, 89-116.

Okamoto, H., Imamura, N., Karppinen, M., Yamauchi, H. \& Fjellvåg, H. (2010). Inorg. Chem. 49, 8709-8712.

Okamoto, H., Karppinen, M., Yamauchi, H. \& Fjellvåg, H. (2009). Solid State Sci. 11, 1211-1215.

Ovsyannikov, S. V., Zainulin, Y. G., Kadyrova, N. I., Tyutyunnik, A. P., Semenova, A. S., Kasinathan, D., Tsirlin, A. A., Miyajima, N. \& Karkin, A. E. (2013). Inorg. Chem. 52, 11703-11710.

Perez-Mato, J. M., Orobengoa, D. \& Aroyo, M. I. (2010). Acta Cryst. A66, 558-590.

Perks, N. J., Johnson, R. D., Martin, C., Chapon, L. C. \& Radaelli, P. G. (2012). Nat. Commun. 3, 1277.

Prodi, A., Daoud-Aladine, A., Gozzo, F., Schmitt, B., Lebedev, O., van Tendeloo, G., Gilioli, E., Bolzoni, F., Aruga-Katori, H., Takagi, H., Marezio, M. \& Gauzzi, A. (2014). Phys. Rev. B, 90, 180101.

Prodi, A., Gilioli, E., Gauzzi, A., Licci, F., Marezio, M., Bolzoni, F., Huang, Q., Santoro, A. \& Lynn, J. W. (2004). Nat. Mater. 3, 4852.

Propach, V. (1977). Z. Anorg. Allg. Chem. 435, 161-171.

Przenioslo, R., Sosnowska, I., Suard, E., Hewat, A. \& Fitch, A. N. (2002). J. Phys. Condens. Matter, 14, 5747-5753.

Rubel, M. H. K., Miura, A., Takei, T., Kumada, N., Mozahar Ali, M., Nagao, M., Watauchi, S., Tanaka, I., Oka, K., Azuma, M., Magome, E., Moriyoshi, C., Kuroiwa, Y. \& Azharul Islam, A. K. M. (2014). Angew. Chem. 126, 3673-3677.

Rubel, M. H. K., Takei, T., Kumada, N., Ali, M. M., Miura, A., Tadanaga, K., Oka, K., Azuma, M., Yashima, M., Fujii, K., Magome, E., Moriyoshi, C., Kuroiwa, Y., Hester, J. R. \& Avdeev, M. (2016). Chem. Mater. 28, 459-465.

Saito, T., Yamada, R., Ritter, C., Senn, M. S., Attfield, J. P. \& Shimakawa, Y. (2014). Inorg. Chem. 53, 1578-1584.

Sakai, Y., Yang, J., Yu, R., Hojo, H., Yamada, I., Miao, P., Lee, S., Torii, S., Kamiyama, T., Ležaić, M., Bihlmayer, G., Mizumaki, M., Komiyama, J., Mizokawa, T., Yamamoto, H., Nishikubo, T., Hattori, Y., Oka, K., Yin, Y., Dai, J., Li, W., Ueda, S., Aimi, A., Mori, D., Inaguma, Y., Hu, Z., Uozumi, T., Jin, C., Long, Y. \& Azuma, M. (2017). J. Am. Chem. Soc. 139, 4574-4581.

Sakhnenko, V. P., Talanov, V. M. \& Chechin, G. M. (1986). Phys. Met. Metallogr. 62, 10-18.

Sales, B. C., Mandrus, D. \& Williams, R. K. (1996). Science, 272, 1325 1328.

Sato, M. \& Hama, Y. (1993). J. Mater. Chem. 3, 233-236.

Schoenflies, A. (1891). Kristallsysteme und Kristallstruktur. Leipzig.

Senn, M. S. \& Bristowe, N. C. (2018). Acta Cryst. A74, 308-321.

Senn, M. S., Chen, W., Saito, T., García-Martín, S., Attfield, J. P. \& Shimakawa, Y. (2014). Chem. Mater. 26, 4832-4837.

Shimakawa, Y. (2008). Inorg. Chem. 47, 8562-8570.

Shimakawa, Y., Zhang, S., Saito, T., Lufaso, M. W. \& Woodward, P. M. (2014). Inorg. Chem. 53, 594-599.

Shimura, G., Niwa, K., Shirako, Y., Muto, M., Kusaba, K. \& Hasegawa, M. (2016). Solid State Commun. 234-235, 40-44.

Shiraki, H., Saito, T., Yamada, T., Tsujimoto, M., Azuma, M., Kurata, H., Isoda, S., Takano, M. \& Shimakawa, Y. (2007). Phys. Rev. B, 76, 140403 .

Shiro, K., Yamada, I., Ikeda, N., Ohgushi, K., Mizumaki, M., Takahashi, R., Nishiyama, N., Inoue, T. \& Irifune, T. (2013). Inorg. Chem. 52, 1604-1609.

Shirokov, V. B. \& Torgashev, V. I. (2004). Crystallogr. Rep. 49, 20-28. Sinclair, D. C., Adams, T. B., Morrison, F. D. \& West, A. R. (2002). Appl. Phys. Lett. 80, 2153-2155.

Sławiński, W. A., Okamoto, H. \& Fjellvåg, H. (2017). Acta Cryst. B73, 313-320.

Sławiński, W., Przeniosło, R., Sosnowska, I. \& Bieringer, M. (2010). J. Phys. Condens. Matter, 22, 186001. 
Sławiński, W., Przeniosło, R., Sosnowska, I., Bieringer, M., Margiolaki, I. \& Suard, E. (2009). Acta Cryst. B65, 535-542.

Stokes, H. T. \& Campbell, B. J. (2017). Acta Cryst. A73, 4-13.

Stokes, H. T. \& Hatch, D. M. (1988). Isotropy Subgroups of the 230 Crystallographic Space Groups. Singapore: World Scientific.

Stokes, H. T. \& Hatch, D. M. (2002). J. Appl. Cryst. 35, 379.

Stokes, H. T., Kisi, E. H., Hatch, D. M. \& Howard, C. J. (2002). Acta Cryst. B58, 934-938.

Streltsov, S. V. \& Khomskii, D. I. (2014). Phys. Rev. B, 89, 201115.

Subramanian, M. A., Li, D., Duan, N., Reisner, B. A. \& Sleight, A. W. (2000). J. Solid State Chem. 151, 323-325.

Takata, K., Yamada, I., Azuma, M., Takano, M. \& Shimakawa, Y. (2007). Phys. Rev. B, 76, 024429.

Talanov, M. V. (2018). Cryst. Growth Des. 18, 3433-3440.

Talanov, M. V., Shirokov, V. B., Avakyan, L. A., Talanov, V. M. \& Borlakov, Kh. Sh. (2018). Acta Cryst. B74, 337-353.

Talanov, M. V., Shirokov, V. B. \& Talanov, V. M. (2014). Crystallogr. Rep. 59, 662-678.

Talanov, M. V., Shirokov, V. B. \& Talanov, V. M. (2016). Acta Cryst. A72, 222-235.

Talanov, V. M. (2007). Glass Phys. Chem. 33, 620-635.

Talanov, V. M. \& Shirokov, V. B. (2014). Acta Cryst. A70, 49-63.

Talanov, V. M., Shirokov, V. B. \& Talanov, M. V. (2015). Acta Cryst. A71, 301-318.

Talanov, V. M., Talanov, M. V. \& Shirokov, V. B. (2014). Crystallogr. Rep. 59, 650-661.

Thomas, N. W. \& Beitollahi, A. (1994). Acta Cryst. B50, 549-560.

Tilley, R. J. D. (2016). Perovskites: Structure-Property Relationships. New York: John Wiley \& Sons.

Tohyama, T., Senn, M. S., Saito, T., Chen, W.-T., Tang, C. C., Attfield, J. P. \& Shimakawa, Y. (2013). Chem. Mater. 25, 178-183.

Toledano, J. C. \& Toledano, P. (1987). The Landau Theory of Phase Transitions. Singapore: World Scientific.

Toledano, P. \& Dmitriev, V. V. (1996). Reconstructive Phase Transitions: in Crystals and Quasicrystals. Singapore: World Scientific.

Torgashev, V. I., Shirokov, V. B., Prokhorov, A. S. \& Shuvalov, L. A. (2005). Crystallogr. Rep. 50, 637-645.

Toyoda, M., Saito, T., Yamauchi, K., Shimakawa, Y. \& Oguchi, T. (2015). Phys. Rev. B, 92, 014420.

Troyanchuk, I. O. \& Chobot, A. N. (1997). Crystallogr. Rep. 42, $983-$ 989.

Tselev, A., Brooks, C. M., Anlage, S. M., Zheng, H., Salamanca-Riba, L., Ramesh, R. \& Subramanian, M. A. (2004). Phys. Rev. B, 70, 144101.

Vasil'ev, A. N. \& Volkova, O. S. (2007). Low Temp. Phys. 33, 895-914.

Vinberg, E. B., Gufan, Yu. M., Sakhnenko, V. P. \& Sirotin, Y. I. (1974). Kristallografiya, 19, 21-26.

Wang, X., Chai, Y., Zhou, L., Cao, H., Cruz, C., Yang, J., Dai, J., Yin, Y., Yuan, Z., Zhang, S., Yu, R., Azuma, M., Shimakawa, Y., Zhang,
H., Dong, S., Sun, Y., Jin, C. \& Long, Y. (2015). Phys. Rev. Lett. 115, 087601.

Wei, W., Li, W., Butler, K. T., Feng, G., Howard, C. J., Carpenter, M. A., Lu, P., Walsh, A. \& Cheetham, A. K. (2018). Angew. Chem. Int. Ed. 57, 8932-8936.

Woodward, P. M. (1997a). Acta Cryst. B53, 32-43.

Woodward, P. M. (1997b). Acta Cryst. B53, 44-66.

Wu, Y., Binford, T., Hill, J. A., Shaker, S., Wang, J. \& Cheetham, A. K. (2018). Chem. Commun. 54, 3751-3754.

Yagi, S., Yamada, I., Tsukasaki, H., Seno, A., Murakami, M., Fujii, H., Chen, H., Umezawa, N., Abe, H., Nishiyama, N. \& Mori, S. (2015). Nat. Commun. 6, 8249.

Yamada, I. (2017). Sci. Technol. Adv. Mater. 18, 541-548.

Yamada, I., Etani, H., Tsuchida, K., Marukawa, S., Hayashi, N., Kawakami, T., Mizumaki, M., Ohgushi, K., Kusano, Y., Kim, Y., Tsuji, N., Takahashi, R., Nishiyama, N., Inoue, T., Irifune, T. \& Takano, M. (2013). Inorg. Chem. 52, 13751-13761.

Yamada, I., Murakami, M., Hayashi, N. \& Mori, S. (2016). Inorg. Chem. 55, 1715-1719.

Yamada, I., Takata, K., Hayashi, N., Shinohara, S., Azuma, M., Mori, S., Muranaka, S., Shimakawa, Y. \& Takano, M. (2008). Angew. Chem. Int. Ed. 47, 7032-7035.

Yamada, I., Tsuchida, K., Ohgushi, K., Hayashi, N., Kim, J., Tsuji, N., Takahashi, R., Matsushita, M., Nishiyama, N., Inoue, T., Irifune, T., Kato, K., Takata, M. \& Takano, M. (2011). Angew. Chem. Int. Ed. 50, 6579-6582.

Yao, W., Guo, Y.-Y. \& Lightfoot, P. (2017). Dalton Trans. 46, 13349 13351.

Zhang, G., Dong, S., Yan, Z., Guo, Y., Zhang, Q., Yunoki, S., Dagotto, E. \& Liu, J.-M. (2011a). Phys. Rev. B, 84, 174413.

Zhang, G., Li, G., Huang, F., Liao, F., Li, K., Wang, Y. \& Lin, J. (2011b). J. Alloys Compd. 509, 9804-9808.

Zhang, L., Terada, N., Johnson, R. D., Khalyavin, D. D., Manuel, P., Katsuya, Y., Tanaka, M., Matsushita, Y., Yamaura, K. \& Belik, A. A. (2018). Inorg. Chem. 57, 5987-5998.

Zhang, S., Saito, T., Chen, W.-T., Mizumaki, M. \& Shimakawa, Y. (2013). Inorg. Chem. 52, 10610-10614.

Zhang, S., Saito, T., Mizumaki, M., Chen, W.-T., Tohyama, T. \& Shimakawa, Y. (2013). J. Am. Chem. Soc. 135, 6056-6060.

Zhang, S., Saito, T., Mizumaki, M. \& Shimakawa, Y. (2014). Chem. Eur. J. 20, 9510-9513.

Zhou, L., Dai, J., Chai, Y., Zhang, H., Dong, S., Cao, H., Calder, S., Yin, Y., Wang, X., Shen, X., Liu, Z., Saito, T., Shimakawa, Y., Hojo, H., Ikuhara, Y., Azuma, M., Hu, Z., Sun, Y., Jin, C. \& Long, Y. (2017). Adv. Mater. 29, 1703435.

Zhu, Y., Zheng, J. C., Wu, L., Frenkel, A. I., Hanson, J., Northrup, P. \& Ku, W. (2007). Phys. Rev. Lett. 99, 037602.

Zubkova, N. V., Arakcheeva, A. V., Pushcharovskii, D. Yu., Semenov, E. I. \& Atencio, D. (2000). Crystallogr. Rep. 45, 210-214. 